\title{
NEW CONCENTRATION PHENOMENA FOR A CLASS OF RADIAL FULLY NONLINEAR EQUATIONS
}

\author{
Giulio GALISE, Alessandro IACOPETTI, Fabiana LEONI, Filomena PACELLA
}

\begin{abstract}
We study radial sign-changing solutions of a class of fully nonlinear elliptic Dirichlet problems in a ball, driven by the extremal Pucci's operators and with a power nonlinear term. We first determine a new critical exponent related to the existence or nonexistence of such solutions. Then we analyze the asymptotic behavior of the radial nodal solutions as the exponents approach the critical values, showing that new concentration phenomena occur. Finally we define a suitable weighted energy for these solutions and compute its limit value.
\end{abstract}

\section{INTRODUCTION}

Let $B$ be the unit ball of $\mathbb{R}^{N}$ and let $0<\lambda \leq \Lambda$. We consider the problem

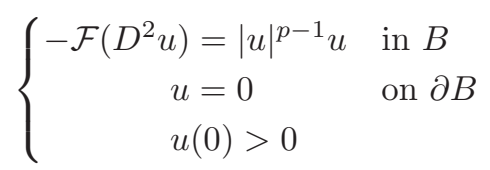

where $p>1, \mathcal{F}$ is either one of the Pucci's extremal operators $\mathcal{M}_{\lambda, \Lambda}^{ \pm}$, defined respectively as

$$
\begin{aligned}
& \mathcal{M}_{\lambda, \Lambda}^{-}(X):=\inf _{\lambda I \leq A \leq \Lambda I} \operatorname{tr}(A X)=\lambda \sum_{\mu_{i}>0} \mu_{i}+\Lambda \sum_{\mu_{i}<0} \mu_{i} \\
& \mathcal{M}_{\lambda, \Lambda}^{+}(X):=\sup _{\lambda I \leq A \leq \Lambda I} \operatorname{tr}(A X)=\Lambda \sum_{\mu_{i}>0} \mu_{i}+\lambda \sum_{\mu_{i}<0} \mu_{i},
\end{aligned}
$$

$\mu_{1}, \ldots, \mu_{N}$ being the eigenvalues of any squared symmetric matrix $X$.

Obviously when $\lambda=\Lambda$, (1.1) is the classical Lane-Emden problem, because Pucci's operators reduce to a multiple of the Laplacian.

Let us immediately observe that, since $\mathcal{M}_{\lambda, \Lambda}^{+}(-X)=-\mathcal{M}_{\lambda, \Lambda}^{-}(X)$, solutions of (1.1) for $\mathcal{F}=\mathcal{M}_{\lambda, \Lambda}^{-}$are solutions of the analogous problem for the operator $\mathcal{F}=\mathcal{M}_{\lambda, \Lambda}^{+}$, but with $u(0)<0$. Thus, it is important to fix the sign at the center of the ball.

The study of (1.1), apart from being interesting in itself, is important to understand some invariance of the Pucci's operators which may be not so evident by their definition.

Indeed, though (1.1) does not have a variational structure when $\lambda<\Lambda$ (as it happens instead for the classical Lane-Emden problem) some critical exponents appear in connection with the existence of solutions. For positive solutions (which are radial by the symmetry result of [6]) they are related to the existence of radial fast decaying solutions of the analogous problem in $\mathbb{R}^{N}$ (see [9]) and induce a concentration phenomenon for positive solutions of (1.1), as $p$ approaches

2010 Mathematics Subject Classification. 35J60; 35B50; 34 B15.

Key words and phrases. Fully nonlinear Dirichlet problems; radial solutions; critical exponents; sign-changing solutions; asymptotic analysis.

Acknowledgements. This research is partially supported by INDAM-GNAMPA . 
the critical values (see 4]). Moreover a weighted related "energy" was defined in [4] which is preserved in the limit, thought the positive solutions concentrate at the origin and converge to zero everywhere else.

The aim of the present paper is to study the asymptotic behavior of radial sign-changing solutions of (1.1) as the exponent $p$ approaches some critical values for their existence. We will show that new critical exponents and new concentration phenomena occur, quite different from those related to the classical Lane-Emden problem but also different from those shown in 4 for the positive solutions of (1.1).

First of all we prove that a new critical exponent $p_{+}^{* *}$ appears for the existence of radial nodal solutions to (1.1) when $\mathcal{F}=\mathcal{M}_{\lambda, \Lambda}^{+}$, which is in between those for the existence of radial positive solutions for the two Pucci's operators (see (1.4)). This is somehow surprising because, since the solutions of (1.1) are positive in the first nodal region, which is a ball, one would expect the critical exponents to be the same as the one for positive solutions to (1.1). Indeed this is the case for $\mathcal{F}=\mathcal{M}_{\lambda, \Lambda}^{-}$and for the classical Laplacian, but not for $\mathcal{F}=\mathcal{M}_{\lambda, \Lambda}^{+}$(see Theorem 1.1).

Then we perform an accurate asymptotic analysis of radial nodal solutions of (1.1) with any number $k$ of nodal domains and show that the behavior can be different in each nodal region and may also depend on $k$ being even or odd (see Theorem 1.2 and Theorem 1.4). Indeed while in some nodal domain there is blow up and concentration in others the solutions are bounded and converge to a finite limit. Moreover the asymptotic profile of the solutions $u$ of (1.1), after suitable rescalings, can be different and, in the case of $\mathcal{F}=\mathcal{M}_{\lambda, \Lambda}^{+}$, the fast decaying radial positive solution of (1.12) in the exterior of the ball appears as limit profile of the restriction of $u$ to some nodal regions (see Proposition 7.3).

This is a completely new phenomenon, to our knowledge, different from what happens for the classical Lane-Emden problem (see [7] and the references therein) and even from what happens in the case of the classical Brezis-Nirenberg problem in low-dimensions which also presents some peculiar asymptotic behavior (see [1, [2, [12, 13, 14, 15).

Finally, all this reflects into the computation of the limit of some weighted energies which can be defined for solutions of (1.1), according to what done in 4, even if (1.1) does not have a variational structure.

We will show that the weighted energy of the positive fast decaying solutions, both in $\mathbb{R}^{N}$ and in $\mathbb{R}^{N} \backslash B$ will contribute to the limit of the total energy of $u$ in some of the nodal regions where blow up and concentration occur.

To state precisely our results let us start by recalling what is known for positive solutions to (1.1).

In the paper [9] Felmer and Quaas proved that there exist two critical exponents $p_{-}^{*}, p_{+}^{*}$ such that positive radial classical solutions to (1.1) exist if and only if $p<p_{-}^{*}$ for $\mathcal{F}=\mathcal{M}_{\lambda, \Lambda}^{-}$or $p<p_{+}^{*}$ when $\mathcal{F}=\mathcal{M}_{\lambda, \Lambda}^{+}$. We observe that the values of these critical exponents are not explicitly known but they satisfy the following inequalities:

$$
\begin{array}{r}
\frac{\tilde{N}_{-}+2}{\tilde{N}_{-}-2}<p_{-}^{*}<\frac{N+2}{N-2}, \\
\max \left\{\frac{\tilde{N}_{+}}{\tilde{N}_{+}-2}, \frac{N+2}{N-2}\right\}<p_{+}^{*}<\frac{\tilde{N}_{+}+2}{\tilde{N}_{+}-2},
\end{array}
$$

where the dimension-like parameters $\tilde{N}_{ \pm}$are defined, respectively by $\tilde{N}_{-}:=\frac{\Lambda}{\lambda}(N-1)+1$, $\tilde{N}_{+}:=\frac{\lambda}{\Lambda}(N-1)+1$. 
We point out that in the special case $\lambda=\Lambda$, when $\mathcal{M}_{\lambda, \Lambda}^{-}=\mathcal{M}_{\lambda, \Lambda}^{+}=\lambda \Delta$, where $\Delta$ is the standard Laplacian, all the above inequalities become equalities. In particular $p_{-}^{*}, p_{+}^{*}$ reduce to the usual Sobolev critical exponent $\frac{N+2}{N-2}$.

In this paper we will always assume that $\lambda<\Lambda$ and $\tilde{N}_{ \pm}>2$.

As far as the existence of radial sign-changing solutions is concerned, let us mention that in [10] a sufficient condition on the exponent $p$ is provided for general radially symmetric nonlinear operators which, in the particular case of the Pucci's operators, reads as $p \leq \frac{\tilde{N}_{-}}{\tilde{N}_{-}-2}$.

The first result of the present paper shows that such a bound on the exponent $p$ is not optimal. Indeed we get:

Theorem 1.1. We have:

i) if $\mathcal{F}=\mathcal{M}_{\lambda, \Lambda}^{-}$, then radial sign-changing solutions of (1.1) with any number of nodal domains exist if and only if

$$
p<p_{-}^{*}
$$

ii) if $\mathcal{F}=\mathcal{M}_{\lambda, \Lambda}^{+}$, then there exists a new critical exponent $p_{+}^{* *}$ satisfying

$$
p_{-}^{*}<p_{+}^{* *}<p_{+}^{*},
$$

such that no radial sign-changing solutions to (1.1) exist for $p \geq p_{+}^{* *}$, while radial signchanging solutions to (1.1) with any number of nodal domains exist at least for a sequence of exponents $p_{n} \nearrow p_{+}^{* *}$.

The above result will be proved in Section 3. Let us observe that while it is easy to obtain i), using Theorem 3.1 of [11, the proof of ii) is quite involved and requires several steps.

Once we have these critical exponents we proceed studying the asymptotic behavior of the nodal solutions of (1.1) as $p$ approaches them to determine also their limit profile. As announced before, we will see that new concentration phenomena occur.

We first start by analyzing the case when $\mathcal{F}=\mathcal{M}_{\lambda, \Lambda}^{-}$. Let $p_{\varepsilon}:=p_{-}^{*}-\varepsilon$, where $\varepsilon>0$ is a small parameter and let us consider the problem

$$
\left\{\begin{array}{cl}
-\mathcal{M}_{\lambda, \Lambda}^{-}\left(D^{2} u\right)=|u|^{p_{\varepsilon}-1} u & \text { in } B \\
u=0 & \text { on } \partial B \\
u(0)>0 &
\end{array}\right.
$$

Let $u_{\varepsilon}$ be a radial sign-changing solution of (1.5) with $k \geq 2$ nodal regions. We denote by $r_{1}=r_{1}(\varepsilon)<\ldots<r_{k-1}=r_{k-1}(\varepsilon)$ the nodal radii of $u_{\varepsilon}$ and by $s_{i}=s_{i}(\varepsilon)$ the unique maximum points of $\left|u_{\varepsilon}\right|$ in the $(i+1)$-th nodal region, for $i=0, \ldots, k-1$. We have

$$
0=s_{0}<r_{1}<s_{1}<\ldots<r_{k-1}<s_{k-1}<1,
$$

and we set $M_{i}:=\left|u_{\varepsilon}\left(s_{i}\right)\right|$, for $i=0,1, \ldots, k-1$.

Theorem 1.2. Up to a subsequence, as $\varepsilon \rightarrow 0^{+}$, we have that $M_{0} \rightarrow+\infty, r_{1} \rightarrow 0, s_{1} \rightarrow 0$, $M_{i} \rightarrow \bar{M}_{i} \in(0,+\infty)$, for $i=1, \ldots, k-1$, and $u_{\varepsilon} \rightarrow \bar{u}$ in $C_{l o c}^{2}(\bar{B} \backslash\{0\})$, where $\bar{u}$ is a radial sign-changing solution of

$$
\left\{\begin{array}{cl}
-\mathcal{M}_{\lambda, \Lambda}^{-}\left(D^{2} u\right)=|u|^{p_{-}^{*}-1} u & \text { in } B \\
u=0 & \text { on } \partial B \\
u(0)<0 &
\end{array}\right.
$$


with $(k-1)$ nodal regions, if $k \geq 3$, while $\bar{u}$ is the unique negative solution of (1.6) if $k=2$.

Moreover if $k \geq 3$ we have $r_{i} \rightarrow \bar{r}_{i}, s_{i} \rightarrow \bar{s}_{i}$, for $i=2, \ldots, k-1$, for some numbers $\bar{r}_{i}, \bar{s}_{i}$, such that $0<\bar{r}_{2}<\bar{s}_{2}<\ldots<\bar{r}_{k-1}<\bar{s}_{k-1}<1$.

Note that (1.6) does not admit a positive solution, by (1.2), but it has a (unique) negative solution as well as sign-changing solutions by (1.3), since $u(0)<0$ so that the relevant exponents for the existence of solutions to (1.6) are those for the corresponding equations involving the operator $\mathcal{M}_{\lambda, \Lambda}^{+}$, but requiring the positivity at the origin.

Even if the problems that we are considering do not have a variational structure, we can introduce, in the spirit of [4, a weighted energy $E_{p}^{T}(u)$ defined for radial sign-changing functions $u$ which change concavity only once in each nodal region, and where $p>1$ is a fixed exponent (we refer to Sect. 10 for the definition). In particular, if $u_{\varepsilon}$ is as in the statement of Theorem 1.2 we are interested in determining the limit energy $E_{p_{\varepsilon}}^{T}\left(u_{\varepsilon}\right)$ as $\varepsilon \rightarrow 0^{+}$. To this end, denoting by $U_{-}$the unique (up to scaling) positive radial fast decaying solution of

$$
-\mathcal{M}_{\lambda, \Lambda}^{-}\left(D^{2} u\right)=u^{p_{-}^{*}} \text { in } \mathbb{R}^{N}
$$

and setting

$$
\Sigma_{-}^{*}:=E^{*}\left(U_{-}\right),
$$

where $E^{*}\left(U_{-}\right)$is the (finite) energy of $U_{-}$in $\mathbb{R}^{N}$ with $p=p_{-}^{*}$ (see (10.7)) we have the following.

Theorem 1.3. Let $u_{\varepsilon}$ be as in Theorem 1.2, It holds

$$
\lim _{\varepsilon \rightarrow 0^{+}} E_{p_{\varepsilon}}^{T}\left(u_{\varepsilon}\right)=\Sigma_{-}^{*}+E_{p_{-}^{*}}^{T}(\bar{u}),
$$

where $\Sigma_{-}^{*}$ is defined by (1.7) and $E_{p_{-}^{*}}^{T}(\bar{u})$ is the total energy of the limit function $\bar{u}$ (given by Theorem 1.2), i.e.

$$
E_{p_{-}^{*}}^{T}(\bar{u})=\sum_{j=1}^{k-1} E_{p_{-}^{*}, \Omega^{j}}\left(\bar{u}^{j}\right), \quad j=1, \ldots, k-1,
$$

where $\bar{u}^{j}$ is the restriction of $\bar{u}$ to its $j$-th nodal region $\Omega^{j}, j=1, \ldots, k-1$ and $E_{p_{-}^{*}, \Omega^{j}}\left(\bar{u}^{j}\right)$ is its energy as defined in (10.4).

When $\mathcal{F}=\mathcal{M}_{\lambda, \Lambda}^{+}$the picture is quite different. Setting $p_{n}:=p_{+}^{* *}-\varepsilon_{n}$, where $\varepsilon_{n}>0$ is a sequence converging to zero as $n \rightarrow+\infty$, we consider a radial sign-changing solution $u_{n}$ of the problem

$$
\left\{\begin{array}{cl}
-\mathcal{M}_{\lambda, \Lambda}^{+}\left(D^{2} u\right)=|u|^{p_{n}-1} u & \text { in } B \\
u=0 & \text { on } \partial B \\
u(0)>0 &
\end{array}\right.
$$

As before, for $k \geq 2$ and $i=1, \ldots, k-1$, we denote by $r_{i}=r_{i}(n)$, the nodal radii of $u_{n}$, by $s_{i}=s_{i}(n)$ the unique maximum point in the $(i+1)$-th nodal region and define $M_{i}=\left|u_{n}\left(s_{i}\right)\right|$.

Theorem 1.4. Up to a subsequence, as $n \rightarrow+\infty$, we have:

i) if $k$ is even then $M_{0} \rightarrow+\infty, M_{i} \rightarrow+\infty, r_{i} \rightarrow 0, s_{i} \rightarrow 0$ for all $i=1, \ldots, k-1$, and $u_{n} \rightarrow 0$ in $C_{l o c}^{2}(\bar{B} \backslash\{0\})$. Moreover for $j=0, \ldots, \frac{k-2}{2}$ there exist positive constants $c_{j}$ such that

if $k \geq 4$ we also have that

$$
\frac{M_{2 j}}{M_{2 j+1}} \rightarrow c_{j}
$$

$$
\frac{M_{2 j+1}}{M_{2 j+2}} \rightarrow+\infty,
$$


for $j=0, \ldots, \frac{k-4}{2}$, as $n \rightarrow+\infty$;

ii) if $k$ is odd then $M_{0} \rightarrow+\infty, M_{i} \rightarrow+\infty, r_{i} \rightarrow 0, s_{i} \rightarrow 0$ for all $i=1, \ldots, k-2, r_{k-1} \rightarrow 0$, $s_{k-1} \rightarrow 0, M_{k-1} \rightarrow \bar{M}$, for some $\bar{M}>0$ and $u_{n} \rightarrow \bar{v}$ in $C_{l o c}^{2}(\bar{B} \backslash\{0\})$, where $\bar{v}$ is the unique positive solution of

$$
\left\{\begin{aligned}
-\mathcal{M}_{\lambda, \Lambda}^{+}\left(D^{2} u\right) & =u^{p_{+}^{* *}} & & \text { in } B \\
u & =0 & & \text { on } \partial B .
\end{aligned}\right.
$$

Moreover for $j=0, \ldots, \frac{k-3}{2}$ there exist positive constants $c_{j}$ such that, as $n \rightarrow+\infty$,

$$
\frac{M_{2 j}}{M_{2 j+1}} \rightarrow c_{j}, \quad \frac{M_{2 j+1}}{M_{2 j+2}} \rightarrow+\infty .
$$

To determine the limit energy of $u_{n}$ we denote by $W_{-}$the only positive radial fast decaying solution of

$$
\left\{\begin{array}{cl}
-\mathcal{M}_{\lambda, \Lambda}^{-}\left(D^{2} u\right)=u^{p_{+}^{* *}} & \text { in } \mathbb{R}^{N} \backslash \bar{B} \\
u=0 & \text { on } \partial B
\end{array}\right.
$$

which exists by the results of [11] because $p_{-}^{*}<p_{+}^{* *}$. Then, setting

$$
\Sigma_{+}^{* *}:=E^{* *}\left(W_{-}\right),
$$

where $E^{* *}\left(W_{-}\right)$is the (finite) energy in $\mathbb{R}^{N} \backslash B$ of $W_{-}$(see (10.9)), we have the following.

Theorem 1.5. Let $u_{n}$ be as in the statement of Theorem 1.4. We have

$$
\lim _{n \rightarrow+\infty} E_{p_{n}}^{T}\left(u_{n}\right)= \begin{cases}\frac{k}{2} E_{p_{+}^{* *}, B}(\bar{v})+\frac{k}{2} \Sigma_{+}^{* *} & \text { if } k \text { is even, } \\ \frac{k+1}{2} E_{p_{+}^{* *}, B}(\bar{v})+\frac{k-1}{2} \Sigma_{+}^{* *} & \text { if } k \text { is odd },\end{cases}
$$

where $\Sigma_{+}^{* *}$ is defined by (1.13), and $E_{p_{+}^{* *}, B}(\bar{v})$ is the energy of the only radial positive solution $\bar{v}$ to (1.11) (see (10.2)).

The proofs of the above results are quite involved and combine several methods: blow up techniques and study of some limit problems, phase plane analysis for the corresponding ODE's and estimates on related pointwise energies.

The outline of the paper is the following. In Section 2 we recall some preliminary results on positive solutions. In Section 3 we prove Theorem 1.1. In Section 4 and Section 5 we consider the case of solutions to (1.1) for $\mathcal{F}=\mathcal{M}_{\lambda, \Lambda}^{-}$with two or three nodal regions. This allows to study the case of any number $k$ of nodal domains by induction in Section 6 , proving so Theorem 1.2. In Section 7 we study problem (1.1) for $\mathcal{F}=\mathcal{M}_{\lambda, \Lambda}^{+}$and solutions with two nodal regions, while in Section 8 we consider the case of three nodal domains. The proof of Theorem 1.4 is then presented in Section 9, again by an induction argument.

Finally in Section 10 we study the total energy associated to the nodal solutions of (1.1) and prove Theorem 1.3 and Theorem 1.5 .

\section{Preliminary Results on POSitive RAdial SOlutions}

We begin this section by recalling the known results about the asymptotic analysis of positive radial solutions to

$$
\left\{\begin{aligned}
-\mathcal{F}\left(D^{2} u\right) & =u^{p} & & \text { in } B \\
u & =0 & & \text { on } \partial B
\end{aligned}\right.
$$


as $p$ approaches the critical exponent for which such solutions exist (for the proofs we refer to (4)). We first introduce some notation: let $\varepsilon>0$ be a small parameter and set

$$
p_{\varepsilon}:= \begin{cases}p_{+}^{*}-\varepsilon, & \text { if } \mathcal{F}=\mathcal{M}_{\lambda, \Lambda}^{+}, \\ p_{-}^{*}-\varepsilon, & \text { if } \mathcal{F}=\mathcal{M}_{\lambda, \Lambda}^{-} .\end{cases}
$$

We denote by $v_{p_{\varepsilon}, \pm}$ the unique positive solution of 2.11. Namely $v_{p_{\varepsilon},+}$ is the only positive solution to (2.1) if $\mathcal{F}=\mathcal{M}_{\lambda, \Lambda}^{+}$, and $v_{p_{\varepsilon},-}$ is the only positive solution to (2.1) if $\mathcal{F}=\mathcal{M}_{\lambda, \Lambda}^{-}$. Accordingly, we denote by $r_{0, \pm}=r_{0, \pm}(\varepsilon) \in(0,1)$ the only radius such that $v_{p_{\varepsilon}, \pm}^{\prime \prime}(r)<0$ for $r \in\left[0, r_{0, \pm}\right)$ and $v_{p_{\varepsilon}, \pm}^{\prime \prime}(r)>0$ for $r \in\left(r_{0, \pm}, 1\right)$. Moreover, let $U_{ \pm}$be the unique positive radial solution of

$$
-\mathcal{M}_{\lambda, \Lambda}^{ \pm}\left(D^{2} u\right)=u^{p_{ \pm}^{*}} \quad \text { in } \mathbb{R}^{N}
$$

such that $U_{ \pm}(0)=1$, and denote by $R_{0, \pm}$ the unique radius such that $U_{ \pm}^{\prime \prime}(r)<0$ for $r \in\left[0, R_{0, \pm}\right)$, $U_{ \pm}^{\prime \prime}(r)>0$ for $r \in\left(R_{0}^{ \pm},+\infty\right)$. We refer to the solutions of (2.2), and in particular to $U_{ \pm}$, as the fast decaying solutions, since for all $p \geq p_{ \pm}^{*}$ and among all radial positive solutions of

$$
-\mathcal{M}_{\lambda, \Lambda}^{ \pm}\left(D^{2} u\right)=u^{p} \quad \text { in } \mathbb{R}^{N}
$$

one has

$$
\lim _{r \rightarrow+\infty} r^{\frac{2}{p-1}} u(r)=0 \Longleftrightarrow p=p_{ \pm}^{*} .
$$

Proposition 2.1. Let $v_{p_{\varepsilon}, \pm}$ be the unique positive solution to (2.1). Then:

(i) $\lim _{\varepsilon \rightarrow 0^{+}}\left\|v_{p_{\varepsilon}, \pm}\right\|_{\infty}=\lim _{\varepsilon \rightarrow 0^{+}} v_{p_{\varepsilon}, \pm}(0)=+\infty$;

(ii) $v_{p_{\varepsilon}, \pm} \rightarrow 0$ in $C_{l o c}^{2}(\bar{B} \backslash\{0\})$, as $\varepsilon \rightarrow 0^{+}$;

(iii) $\lim _{\varepsilon \rightarrow 0^{+}}\left[r_{0, \pm}(\varepsilon)\right]^{\frac{2}{p_{\varepsilon}-1}}\left\|v_{p_{\varepsilon}, \pm}\right\|_{\infty}=\left(R_{0, \pm}\right)^{\frac{2}{p_{ \pm}^{-1}}}$;

(iv) $\lim _{\varepsilon \rightarrow 0^{+}} \frac{v_{p_{\varepsilon}, \pm}\left(r_{0, \pm}(\varepsilon)\right)}{\left\|v_{p_{\varepsilon}, \pm}\right\|_{\infty}}=U_{ \pm}\left(R_{0, \pm}\right)$;

(v) $\lim _{\varepsilon \rightarrow 0^{+}}\left[r_{0, \pm}(\varepsilon)\right]^{\frac{2}{p_{\varepsilon}-1}} v_{p_{\varepsilon}, \pm}\left(r_{0, \pm}(\varepsilon)\right)=\left(R_{0, \pm}\right)^{\frac{2}{p_{ \pm}^{2-1}}} U_{ \pm}\left(R_{0, \pm}\right)$; (vi) $\lim _{\varepsilon \rightarrow 0^{+}}\left\|v_{p_{\varepsilon}, \pm}\right\|_{\infty}^{\frac{p_{\varepsilon}\left(\tilde{N}_{ \pm}-2\right)-\tilde{N}_{ \pm}}{2}}\left(v_{p_{\varepsilon}, \pm}\right)^{\prime}(1)=-C_{ \pm}$, where $C_{ \pm}$is a positive constant depending
only on $N, \lambda, \Lambda$.

Next we recall some useful results about the qualitative properties of the solutions of a suitable class of initial value problems. To do this we need some preliminaries.

If $u$ is a smooth radially symmetric function, we easily check that the Hessian of $u$ is given by

$$
D^{2} u(x)=\frac{u^{\prime}(|x|)}{|x|} \mathbb{I}_{N}+\left(u^{\prime \prime}(|x|)-\frac{u^{\prime}(|x|)}{|x|}\right) \frac{x}{|x|} \otimes \frac{x}{|x|},
$$

where $\mathbb{I}_{N}$ is the identity matrix of order $N$ and $x \otimes x$ is the matrix defined by $(x \otimes x)_{i j}=x_{i} x_{j}$, for any $i, j \in\{1, \ldots, N\}$. In particular, since the eigenvalues of the matrix appearing in the right-hand side of (2.3) are $u^{\prime \prime}(|x|)$, which is simple, and $\frac{u^{\prime}(|x|)}{|x|}$, which has multiplicity $(N-1)$, we infer that if $u$ is a positive radial solution of $-\mathcal{F}\left(D^{2} u\right)=u^{p}$, then setting $r=|x|$ there are only three possibilities:

Case 1: $u^{\prime}(r) \geq 0$ and $u^{\prime \prime}(r) \leq 0$, so that $u=u(r)$ satisfies

$$
\begin{cases}-\Lambda u^{\prime \prime}(r)-\lambda(N-1) \frac{u^{\prime}(r)}{r}=u^{p}(r) & \text { if } \mathcal{F}=\mathcal{M}_{\lambda, \Lambda}^{-}, \\ -\lambda u^{\prime \prime}(r)-\Lambda(N-1) \frac{u^{\prime}(r)}{r}=u^{p}(r) & \text { if } \mathcal{F}=\mathcal{M}_{\lambda, \Lambda}^{+} .\end{cases}
$$


Case 2: $u^{\prime}(r) \leq 0$ and $u^{\prime \prime}(r) \leq 0$, so that $u=u(r)$ satisfies

$$
\begin{cases}-\Lambda\left(u^{\prime \prime}(r)+(N-1) \frac{u^{\prime}(r)}{r}\right)=u^{p}(r) & \text { if } \mathcal{F}=\mathcal{M}_{\lambda, \Lambda}^{-}, \\ -\lambda\left(u^{\prime \prime}(r)+(N-1) \frac{u^{\prime}(r)}{r}\right)=u^{p}(r) & \text { if } \mathcal{F}=\mathcal{M}_{\lambda, \Lambda}^{+} .\end{cases}
$$

Case 3: $u^{\prime}(r) \leq 0$ and $u^{\prime \prime}(r) \geq 0$, so that $u=u(r)$ satisfies

$$
\begin{cases}-\lambda u^{\prime \prime}(r)-\Lambda(N-1) \frac{u^{\prime}(r)}{r}=u^{p}(r) & \text { if } \mathcal{F}=\mathcal{M}_{\lambda, \Lambda}^{-}, \\ -\Lambda u^{\prime \prime}(r)-\lambda(N-1) \frac{u^{\prime}(r)}{r}=u^{p}(r) & \text { if } \mathcal{F}=\mathcal{M}_{\lambda, \Lambda}^{+} .\end{cases}
$$

We stress that the case $u^{\prime}(r) \geq 0$ and $u^{\prime \prime}(r) \geq 0$ cannot occur because $u>0$ satisfies $-\mathcal{F}\left(D^{2} u\right)=$ $u^{p}$.

Now, let $\alpha>0, p>1$ and consider the following initial value problem

$$
\left\{\begin{array}{rlrl}
u^{\prime \prime}(r)= & \left.M_{-}\left(-\frac{\Lambda(N-1)}{r} K_{-}\left(u^{\prime}(r)\right)-u^{p}(r)\right)\right) & \text { for } r>1 \\
& u(r)>0 & & \text { for } r>1 \\
& u(1)=0, \quad u^{\prime}(1)=\alpha &
\end{array}\right.
$$

with

$$
M_{-}(\xi):=\left\{\begin{array}{ll}
\xi / \lambda & \text { if } \xi \geq 0 \\
\xi / \Lambda & \text { if } \xi<0
\end{array}, \quad K_{-}(\xi):= \begin{cases}\frac{\lambda}{\Lambda} \xi & \text { if } \xi \geq 0 \\
\xi & \text { if } \xi<0 .\end{cases}\right.
$$

Problem (2.7) has a unique solution $u_{\alpha}=u(\alpha, p, r)$, defined and positive on a maximal interval $\left[1, \rho_{\alpha}\right)$, for some $1<\rho_{\alpha} \leq+\infty$. In [10] it has been proved that there exists $\tau_{\alpha} \in\left(1, \rho_{\alpha}\right)$ such that $u_{\alpha}^{\prime}(r)>0$ for $r \in\left(1, \tau_{\alpha}\right), u_{\alpha}^{\prime}(r)<0$ for $r \in\left(\tau_{\alpha}, \rho_{\alpha}\right)$. Moreover, there exists $\sigma_{\alpha} \in\left(\tau_{\alpha}, \rho_{\alpha}\right)$ such that $u_{\alpha}^{\prime \prime}<0$ in $\left(1, \sigma_{\alpha}\right)$ and $u_{\alpha}^{\prime \prime}>0$ in $\left(\sigma_{\alpha}, \rho_{\alpha}\right)$ (see [9, 11]).

Concerning the asymptotic properties with respect to the parameter $\alpha$, we recall that $\rho_{\alpha} \rightarrow 1$ as $\alpha \rightarrow+\infty$, while $\rho_{\alpha} \rightarrow+\infty$ as $\alpha \rightarrow 0$ (see [10. Proposition 3.2 and Lemma 3.1]). In particular $\rho_{\alpha}<+\infty$ for all sufficiently large $\alpha>0$ and thus we can define the critical slope

$$
\alpha_{-}^{*}=\alpha_{-}^{*}(p):=\inf \left\{\alpha>0 ; \rho_{\alpha}<+\infty\right\} .
$$

We point out that if $\rho_{\alpha}<+\infty$ then $u_{\alpha}\left(\rho_{\alpha}\right)=0$ and $u(x):=u_{\alpha}(|x|)$ is a positive radial solution of

$$
\left\{\begin{array}{cl}
-\mathcal{M}_{\lambda, \Lambda}^{-}\left(D^{2} u\right)=u^{p} & \text { in } A_{1, \rho_{\alpha}} \\
u=0 & \text { on } \partial A_{1, \rho_{\alpha}}
\end{array}\right.
$$

where $A_{1, \rho_{\alpha}}:=\left\{x \in \mathbb{R}^{N} ; 1<|x|<\rho_{\alpha}\right\}$ is the annulus of radii $1, \rho_{\alpha}$, centered at the origin. If $\rho_{\alpha}=+\infty$ then $u(x):=u_{\alpha}(|x|)$ is a positive radial solution of

$$
\left\{\begin{array}{cl}
-\mathcal{M}_{\lambda, \Lambda}^{-}\left(D^{2} u\right)=u^{p} & \text { in } \mathbb{R}^{N} \backslash \bar{B} \\
u=0 & \text { on } \partial B
\end{array}\right.
$$

In [11] it has been proved that (2.9) has positive radial solutions if and only if $p>p_{-}^{*}$ (see [11, Theorem 1.1]). More precisely, we have the following (see [11, Sect. 6 and Theorem 6.2]):

Theorem 2.2. Let $u_{\alpha}$ denote the maximal positive solution of (2.7). One has $\alpha_{-}^{*}(p)>0$ if and only if $p>p_{-}^{*}$ and, for such $p$,

(i) for any $\alpha>\alpha_{-}^{*}(p)$ it holds that $\rho_{\alpha}<+\infty$;

(ii) $\rho_{\alpha_{-}^{*}}=+\infty$ and $u_{\alpha_{-}^{*}}$ is a fast decaying solution of (2.9);

(iii) if $p_{-}^{*}<p \leq \frac{N+2}{N-2}$, then for any $\alpha<\alpha_{-}^{*}(p), u_{\alpha}$ is either a pseudo-slow or a slow decaying solution; 
(iv) if $p>\frac{N+2}{N-2}$, then for any $\alpha<\alpha_{-}^{*}(p), u_{\alpha}$ is a slow decaying solution.

Analogous results hold for $\mathcal{F}=\mathcal{M}_{\lambda, \Lambda}^{+}$, where one considers the initial value problem

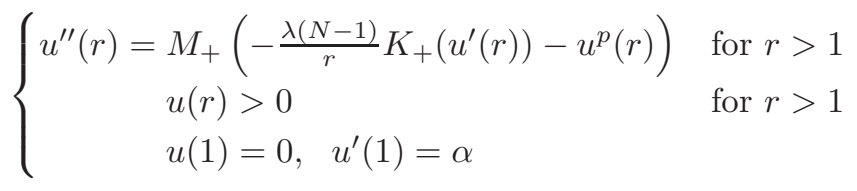

where

$$
M_{+}(\xi):=\left\{\begin{array}{ll}
\xi / \Lambda & \text { if } \xi \geq 0 \\
s / \lambda & \text { if } \xi<0
\end{array}, \quad K_{+}(\xi):=\left\{\begin{array}{ll}
\frac{\Lambda}{\lambda} \xi & \text { if } \xi \geq 0 \\
\xi & \text { if } \xi<0
\end{array} .\right.\right.
$$

We refer to [11] for the precise statements.

We conclude this section by proving a crucial property of the map $p \mapsto \alpha_{-}^{*}(p)$.

Proposition 2.3. The map $p \mapsto \alpha_{-}^{*}(p)$ is continuous in $(1,+\infty)$.

Proof. By Theorem 2.2, one has $\alpha_{-}^{*}(p) \equiv 0$ for $p \in\left(1, p_{-}^{*}\right]$.

Let us first prove that $\alpha_{-}^{*}(p) \rightarrow 0$ for $p \searrow p_{-}^{*}$. By contradiction, assume that there exist $\alpha_{0}>0$ and a sequence $p_{n} \searrow p_{-}^{*}$ such that $\alpha_{-}^{*}\left(p_{n}\right)>\alpha_{0}$ for all $n \in \mathbb{N}$. This means that, for all $n \in \mathbb{N}$, the initial value problem

$$
\left\{\begin{array}{c}
u^{\prime \prime}(r)=M_{-}\left(-\frac{\Lambda(N-1)}{r} K_{-}\left(u^{\prime}(r)\right)-u^{p_{n}}(r)\right) \text { for } r>1 \\
u(1)=0, u^{\prime}(1)=\alpha_{0}
\end{array}\right.
$$

has a solution $u_{n}$ defined and positive in the whole interval $(1,+\infty)$. Let us denote by $s_{n} \in$ $(1,+\infty)$ the unique maximum point of $u_{n}$ and set $m_{n}:=u_{n}\left(s_{n}\right)$. By (2.4), the energy-like functionals

$$
H_{\Lambda, n}(r):=\frac{\left(u_{n}^{\prime}(r)\right)^{2}}{2}+\frac{\left(u_{n}(r)\right)^{p_{n}+1}}{\Lambda\left(p_{n}+1\right)}
$$

are nonincreasing in $\left[1, s_{n}\right]$. Hence, we deduce

$$
m_{n}^{p_{n}+1} \leq \frac{\Lambda\left(p_{n}+1\right)}{2} \alpha_{0}^{2}
$$

Then, $\left(m_{n}\right)_{n}$ is bounded and, from (2.11), we infer that $u_{n} \rightarrow \bar{u}$ in $C_{l o c}^{2}([1,+\infty))$, as $n \rightarrow+\infty$, where $\bar{u}$ is a solution to

$$
\left\{\begin{array}{cc}
\left.u^{\prime \prime}(r)=M_{-}\left(-\frac{\Lambda(N-1)}{r} K_{-}\left(u^{\prime}(r)\right)-u^{p_{-}^{*}}(r)\right)\right) & \text { for } r>1 \\
u \geq 0 & \text { for } r>1 \\
u(1)=0, u^{\prime}(1)=\alpha_{0} . &
\end{array}\right.
$$

Such function $\bar{u}$ cannot be identically zero in view of the initial condition $\bar{u}^{\prime}(1)=\alpha_{0}>0$. Hence, $\bar{u}>0$ in $(1,+\infty)$ and $u(x)=\bar{u}(|x|)$ is a positive radial solution of (2.9) with $p=p_{-}^{*}$, contradicting Theorem 2.2 .

Next, let us show that $\alpha_{-}^{*}$ is continuous in $\left(p_{-}^{*},+\infty\right)$. For any fixed $p_{0}>p_{-}^{*}$, let us consider $\alpha>\alpha_{-}^{*}\left(p_{0}\right)$. Then, denoting by $u=u_{\alpha, p_{0}}$ the unique maximal solution of the initial value problem

$$
\left\{\begin{array}{c}
u^{\prime \prime}(r)=M_{-}\left(-\frac{\Lambda(N-1)}{r} K_{-}\left(u^{\prime}(r)\right)-|u|^{p_{0}-1} u(r)\right) \text { for } r>1 \\
u(1)=0, u^{\prime}(1)=\alpha,
\end{array}\right.
$$

there exists a $\rho_{\alpha}>1$ such that $u_{\alpha, p_{0}}(r)>0$ in $\left(1, \rho_{\alpha}\right), u_{\alpha, p_{0}}\left(\rho_{\alpha}\right)=0$ and $u_{\alpha, p_{0}}<0$ in a right neighborhood of $\rho_{\alpha}$. By continuous dependence on the data, for $p \rightarrow p_{0}$ the corresponding 
maximal solution $u_{\alpha, p}$ is converging to $u_{\alpha, p_{0}}$ in $C_{l o c}^{2}([1,+\infty))$. Hence, $u_{\alpha, p}$ has a first zero close to $\rho_{\alpha}$ for $p$ close to $p_{0}$, meaning that $\alpha_{-}^{*}(p) \leq \alpha$. By the arbitrary choice of $\alpha>\alpha_{-}^{*}\left(p_{0}\right)$, we deduce that $\limsup _{p \rightarrow p_{0}} \alpha_{-}^{*}(p) \leq \alpha_{-}^{*}\left(p_{0}\right)$.

Conversely, let us now consider $0<\alpha<\alpha_{-}^{*}\left(p_{0}\right)$. Then, the maximal solution $u_{\alpha, p_{0}}$ of problem (2.12) is positive in $(1,+\infty)$. Let us prove that, for $p$ close to $p_{0}$, one has $\alpha_{-}^{*}(p) \geq \alpha$. Arguing by contradiction, let us assume that, for a sequence $p_{n} \rightarrow p_{0}$, the corresponding maximal solutions $u_{\alpha, p_{n}}$ satisfy $u_{\alpha, p_{n}}(r)>0$ in $\left(1, \rho_{n}\right)$ and $u_{\alpha, p_{n}}\left(\rho_{n}\right)=0$ for some $\rho_{n}>1$. Again by continuous dependence on the data, one has that $u_{\alpha, p_{n}} \rightarrow u_{\alpha, p_{0}}$ in $C_{l o c}^{2}([1,+\infty))$, so that $\rho_{n} \rightarrow+\infty$. Moreover, for each $n$ there exists $t_{n} \in\left(1, \rho_{n}\right)$ such that $u_{\alpha, p_{n}}^{\prime \prime}(r)<0$ for $r \in\left[1, t_{n}\right)$ and $u_{\alpha, p_{n}}^{\prime \prime}(r)>0$ for $r \in\left(t_{n}, \rho_{n}\right]$, and the sequence $\left(t_{n}\right)_{n}$ is bounded from above and from below away from 1 , since otherwise the function $u_{\alpha, p_{0}}$ would be globally either concave or convex in $(1,+\infty)$. Thus, possibly considering a subsequence, there exists $t_{0}>1$ such that $t_{n} \rightarrow t_{0}$, with $u_{\alpha, p_{0}}^{\prime \prime}(r)<0$ for $r \in\left[1, t_{0}\right)$ and $u_{\alpha, p_{0}}^{\prime \prime}(r)>0$ for $r>t_{0}$. Now, we claim that there exist positive constants $C, K>0$ independent of $n$ such that

$$
u_{\alpha, p_{n}}(r) \leq \frac{C}{\left(r^{2}-t_{n}^{2}+K\right)^{\frac{\tilde{N}_{-}-2}{2}}} \quad \text { for } r \in\left[t_{n}, \rho_{n}\right] .
$$

Indeed, we observe that in the interval $\left[t_{n}, \rho_{n}\right]$, by (2.6), the function $v_{n}=u_{\alpha, p_{n}}$ satisfies

$$
v_{n}^{\prime \prime}+\frac{\tilde{N}_{-}-1}{r} v_{n}^{\prime}+\frac{v_{n}^{p_{n}}}{\lambda}=0 .
$$

Then, considering the energy-like functional $H_{n}:\left[t_{n}, \rho_{n}\right] \rightarrow \mathbb{R}$ defined by

$$
H_{n}(r):=r^{\tilde{N}_{-}}\left[\frac{\left(v_{n}^{\prime}(r)\right)^{2}}{2}+\frac{\tilde{N}_{-}-2}{2 \lambda \tilde{N}_{-}} v_{n}^{p_{n}+1}\right]+\frac{\tilde{N}_{-}-2}{2} r^{\tilde{N}_{-}-1} v_{n}(r) v_{n}^{\prime}(r),
$$

and exploiting (2.14), we see that

$$
H_{n}^{\prime}(r)=\frac{p_{n}\left(\tilde{N}_{-}-2\right)-\left(\tilde{N}_{-}+2\right)}{2 \lambda \tilde{N}_{-}} r^{\tilde{N}_{-}} v_{n}^{p_{n}}(r) v_{n}^{\prime}(r)<0,
$$

where we use the fact that $p_{n} \rightarrow p_{0}$ and $p_{0}>p_{-}^{*}>\frac{\tilde{N}_{-}+2}{\tilde{N}_{-}-2}$. Hence, $H_{n}$ is decreasing and, in particular, we get that

$$
H_{n}(r) \geq H_{n}\left(\rho_{n}\right)=\rho_{n}^{\tilde{N}_{-}} \frac{\left(v_{n}^{\prime}\left(\rho_{n}\right)\right)^{2}}{2}>0, \text { for any } r \in\left[t_{n}, \rho_{n}\right] .
$$

Now, let us consider the auxiliary functional $J_{n}:\left[t_{n}, \rho_{n}\right] \rightarrow \mathbb{R}$ defined by

$$
J_{n}(r):=v_{n}(r)^{-\frac{\tilde{N}_{-}}{N_{-}-2}} \frac{v_{n}^{\prime}(r)}{r} .
$$

Then, exploiting again (2.14) and the definition of $H_{n}$, we easily check that

$$
J_{n}^{\prime}(r)=-\frac{2 \tilde{N}_{-}}{\tilde{N}_{-}-2} v_{n}(r)^{-\frac{2\left(\tilde{N}_{--1}\right)}{\tilde{N}_{-}-2}} r^{-\left(\tilde{N}_{-}+1\right)} H_{n}(r)<0 .
$$

Therefore, $J_{n}$ is monotone decreasing and thus $J_{n}\left(t_{n}\right) \geq J_{n}(r)$ for any $r \in\left[t_{n}, \rho_{n}\right]$. With the help of (2.14), this can be rewritten as

$$
-\frac{v_{n}\left(t_{n}\right)^{p_{n}-\frac{\tilde{N}_{-}}{\hat{N}_{-}-2}}}{\lambda\left(\tilde{N}_{-}-1\right)} \geq v_{n}(r)^{-\frac{\tilde{N}_{-}}{\hat{N}_{-}-2}} \frac{v_{n}^{\prime}(r)}{r} .
$$


Since $v_{n}\left(t_{n}\right)=u_{\alpha, p_{n}}\left(t_{n}\right) \rightarrow u_{\alpha, p_{0}}\left(t_{0}\right)>0$, from the above inequality we deduce that there exists $C_{1}>0$ independent of $n$ such that, for any $r \in\left[t_{n}, \rho_{n}\right]$,

$$
v_{n}(r)^{-\frac{\tilde{N}-}{N_{-}-2}} v_{n}^{\prime}(r) \leq-C_{1} r .
$$

Integrating between $t_{n}$ and $r$ we obtain

$$
v_{n}(r)^{-\frac{2}{N_{-}-2}}-v_{n}\left(t_{n}\right)^{-\frac{2}{N_{-}-2}} \geq \frac{C_{1}}{\tilde{N}_{-}-2}\left(r^{2}-t_{n}^{2}\right) .
$$

Taking into account that $v_{n}\left(t_{n}\right) \rightarrow u_{\alpha, p_{0}}\left(t_{0}\right)>0$ as before, we infer that

$$
v_{n}(r)^{-\frac{2}{N_{-}-2}} \geq \frac{C_{1}}{\tilde{N}_{-}-2}\left(r^{2}-t_{n}^{2}\right)+K_{1},
$$

for some positive constant $K_{1}$ independent of $n$, and this is exactly (2.13).

Letting $n \rightarrow+\infty$ in (2.13), we then obtain

$$
u_{\alpha, p_{0}}(r) \leq \frac{C}{\left(r^{2}-t_{0}^{2}+K\right)^{\frac{\tilde{N}_{-}-2}{2}}} \quad \text { for } r \geq t_{0} .
$$

Since $p_{0}>p_{-}^{*}>\frac{\tilde{N}_{-}}{\tilde{N}_{-}-2}$, it then follows that

$$
\lim _{r \rightarrow+\infty} r^{\frac{2}{p_{0}-1}} u_{\alpha, p_{0}}(r)=0
$$

meaning that $u_{\alpha, p_{0}}$ is a fast decaying solution of problem (2.9) with $p=p_{0}$. Since $\alpha<\alpha_{-}^{*}\left(p_{0}\right)$, this is again a contradiction to Theorem 2.2 Hence, by the arbitrary choice of $\alpha<\alpha_{-}^{*}\left(p_{0}\right)$, we deduce that $\liminf _{p \rightarrow p_{0}} \alpha_{-}^{*}(p) \geq \alpha_{-}^{*}\left(p_{0}\right)$, which finally proves the continuity of $\alpha_{-}^{*}$.

3. Critical exponents for the EXistence of RADial Sign-Changing Solutions in the BALL

In this section we prove Theorem 1.1. Since the proof of ii) requires several steps we start by considering the case $\mathcal{F}=\mathcal{M}_{\lambda, \Lambda}^{+}$, i.e. we consider the problem

$$
\left\{\begin{array}{cl}
-\mathcal{M}_{\lambda, \Lambda}^{+}\left(D^{2} u\right)=|u|^{p-1} u & \text { in } B \\
u=0 & \text { on } \partial B \\
u(0)>0 &
\end{array}\right.
$$

Let us define the following set

$$
\mathcal{A}:=\left\{p \in(1,+\infty): \text { there exists } u_{p} \text { radial sign-changing solution to (3.1) }\right\} .
$$

Remark 3.1. The set $\mathcal{A}$ is nonempty in view of [10, Theorem 1.3]. Moreover, by a trivial scaling argument, it is easy to check that $\mathcal{A}$ coincides with the set of $p \in(1,+\infty)$ for which there exists a nodal solution $u_{p}$ to (3.1) which changes sign exactly once.

As a first result we show a crucial upper bound for $\sup \mathcal{A}$.

Proposition 3.2. It holds that

$$
\sup \mathcal{A}<p_{+}^{*} \text {. }
$$

Proof. We first observe that $\sup \mathcal{A} \leq p_{+}^{*}$, because for $p \geq p_{+}^{*}$ there cannot exist positive radial solutions to 3.1 . 
Now, assume by contradiction that $\sup \mathcal{A}=p_{+}^{*}$. Then we can find a sequence $\left(u_{p_{n}}\right)_{p_{n} \in \mathcal{A}}$ of nodal radial solutions to (3.1), with $p_{n} \nearrow p_{+}^{*}$. In view of Remark 3.1 we can assume without loss of generality that $u_{p_{n}}$ changes sign exactly once. Let us consider the rescaled function

$$
\tilde{u}_{p_{n}}(x)=r_{1}^{\frac{2}{p_{n}-1}} u_{p_{n}}\left(r_{1} x\right), \quad x \in B_{\frac{1}{r_{1}}},
$$

where $r_{1}=r_{1}(n)$ is the node of $u_{p_{n}}$.

By construction, for $x \in B,\left(\tilde{u}_{p_{n}}\right)_{p_{n} \in \mathcal{A}}$ is a sequence of almost critical positive solutions of (3.1). Then, in view of Proposition 2.1 we have $\tilde{u}_{p_{n}}^{\prime}(1) \rightarrow 0$ as $p_{n} \rightarrow p_{+}^{*}$. In addition, by construction, the function $\tilde{u}_{p_{n}}^{-}(x):=r_{1}^{\frac{2}{p_{n}-1}} u_{p_{n}}^{-}\left(r_{1} x\right), x \in A_{1, \frac{1}{r_{1}}}$ is a positive radial solution of

$$
\left\{\begin{array}{cl}
-\mathcal{M}_{\lambda, \Lambda}^{-}\left(D^{2} u\right)=u^{p_{n}} & \text { in } A_{1, \frac{1}{r_{1}}} \\
u=0 & \text { on } \partial A_{1, \frac{1}{r_{1}}} .
\end{array}\right.
$$

In particular, $\tilde{u}_{p_{n}}^{-}=\tilde{u}_{p_{n}}^{-}(r)$ satisfies (2.7) with $\alpha=\alpha\left(p_{n}\right)=\left(\tilde{u}_{p_{n}}^{-}\right)^{\prime}(1) \rightarrow 0$, as $p_{n} \rightarrow p_{+}^{*}$, and we have $\tilde{u}_{p_{n}}^{-}\left(1 / r_{1}\right)=0$.

On the other hand, since $p_{+}^{*}>p_{-}^{*}$ then in view of [11, Theorem 1.1] and Theorem [2.2 we have $\alpha_{-}^{*}\left(p_{+}^{*}\right)>0$ and, from Proposition 2.3, we can find $\alpha_{0}>0$ such that $\alpha_{-}^{*}(p)>\alpha_{0}>0$ for all $p$ in a sufficiently small neighborhood of $p_{+}^{*}$. In particular, for any $\alpha \in\left(0, \alpha_{0}\right)$ and for any $p$ sufficiently close to $p_{+}^{*}$ the unique solution to (2.7) is defined and positive in the whole $(1,+\infty)$, but this contradicts the properties of $\tilde{u}_{p_{n}}^{-}$, namely that $\tilde{u}_{p_{n}}^{-}\left(1 / r_{1}\right)=0$. This gives a contradiction and the proof is complete.

Proposition 3.3. It holds that

$$
\sup \mathcal{A}>p_{-}^{*} .
$$

Proof. In order to prove the result we construct a sign-changing solution $u_{p}$ to (3.1) for $p$ in a sufficiently small right neighborhood of $p_{-}^{*}$.

To this end, let $p \in\left(p_{-}^{*}, p_{+}^{*}\right)$ and as in Sect. 2 we denote by $v_{p,+}$ the unique positive radial solution of

$$
\left\{\begin{array}{cl}
-\mathcal{M}_{\lambda, \Lambda}^{+}\left(D^{2} u\right)=u^{p} & \text { in } B \\
u=0 & \text { on } \partial B .
\end{array}\right.
$$

Since $p_{-}^{*}<p_{+}^{*}$, choosing $0<\delta<\left(p_{+}^{*}-p_{-}^{*}\right)$ we have $I_{\delta}:=\left(p_{-}^{*}, p_{-}^{*}+\delta\right) \subset\left(p_{-}^{*}, p_{+}^{*}\right)$ and $m_{p}:=\max v_{p,+}=v_{p,+}(0)$ is uniformly bounded for $p \in I_{\delta}$. Moreover we can find $\gamma>0$ such that for all $p \in I_{\delta}$ it holds $\left|v_{p,+}^{\prime}(1)\right|>\gamma>0$. Hence, exploiting Proposition 2.3 and since $\alpha_{-}^{*}(p) \rightarrow 0$ as $p \rightarrow p_{-}^{*}$ (see the proof of Proposition 2.3) we find a right neighborhood of $p_{-}^{*}$, let us say $I_{\varepsilon}:=\left(p_{-}^{*}, p_{-}^{*}+\varepsilon\right)$ with $\varepsilon<\delta$, such that $\left|v_{p,+}^{\prime}(1)\right|>\alpha_{-}^{*}(p)$, for all $p \in I_{\varepsilon}$. This means that for all $p \in I_{\varepsilon}$, if we take $\alpha=\alpha(p)=-v_{p,+}^{\prime}(1)$ in (2.7) then we have $\rho_{\alpha}=\rho_{\alpha}(p)<+\infty$ and the unique maximal positive solution $u_{\alpha(p)}=u_{\alpha(p)}(r)$ vanishes at $r=\rho_{\alpha(p)}$. Hence for $p \in I_{\varepsilon}$ we can glue the two solutions by defining $z_{p}:\left[0, \rho_{\alpha(p)}\right] \rightarrow \mathbb{R}$ as

$$
z_{p}(r):= \begin{cases}v_{p,+}(r) & \text { if } r \in[0,1], \\ -u_{\alpha(p)}(r) & \text { if } r \in\left(1, \rho_{\alpha(p)}\right] .\end{cases}
$$

Then, setting $u_{p}(r):=\left[\rho_{\alpha(p)}\right]^{\frac{2}{p-1}} z_{p}\left(r \rho_{\alpha(p)}\right)$ we easily check that $u_{p}(x)=u_{p}(|x|)$ is a radial sign-changing solution to (3.1).

By the very definition of $\sup \mathcal{A}$ we have that for $p>\sup \mathcal{A}$ radial sign-changing solution to (3.1) cannot exist. In the next proposition we show that the same happens for $p=\sup \mathcal{A}$.

Proposition 3.4. It holds that $\sup \mathcal{A} \notin \mathcal{A}$. 
Proof. Assume by contradiction that there exists a a radial sign-changing solution $u_{*}$ to 3.1 for $p=\sup \mathcal{A}$. In view of Remark 3.1 we can assume without loss of generality that $u_{*}$ changes sign exactly once and we denote by $r_{*} \in(0,1)$ its nodal radius.

Let us consider the rescaled radial function $\tilde{u}_{*}(x):=r_{*}^{\frac{2}{p-1}} u_{*}\left(r_{*} x\right), x \in B_{1 / r_{*}}$ and set $\alpha_{*}:=$ $-\tilde{u}_{*}^{\prime}(1)>0$. Then, by construction $\left(\tilde{u}_{*}\right)^{-}(r)$ (the negative part of $\tilde{u}_{*}$, defined by taking the maximum between $-\tilde{u}_{*}$ and zero) coincides for $r \in\left[1,1 / r_{*}\right]$ with the unique maximal positive solution to (2.7) with $\alpha=\alpha_{*}, p=\sup \mathcal{A}$.

Therefore, since $\sup \mathcal{A}>p_{-}^{*}$ and $\left(\tilde{u}_{*}\right)^{-}\left(1 / r_{*}\right)=0$, from Theorem 2.2 we have

$$
\alpha_{*}>\alpha_{-}^{*}(\sup \mathcal{A}) \text {. }
$$

Now we observe that, $\operatorname{since} \sup \mathcal{A}<p_{+}^{*}$, up to a subsequence, as $p_{n} \searrow \sup \mathcal{A}$ the unique positive solution $v_{p_{n},+}$ to (3.4) converges in $C^{2}(\bar{B})$ to $\left.\tilde{u}_{*}\right|_{\bar{B}}$. In particular this implies that $-v_{p_{n},+}^{\prime}(1) \rightarrow \alpha_{*}$, for some sequence $p_{n} \searrow \sup \mathcal{A}$. Moreover, from Proposition 2.3 and (3.5) we infer that $-v_{p_{n},+}^{\prime}(1)>\alpha_{-}^{*}\left(p_{n}\right)$ for all $p_{n}$ sufficiently close to $\sup \mathcal{A}$.

Therefore, fixing $p_{n}>\sup \mathcal{A}$ sufficiently close to $\sup \mathcal{A}$, and taking $\alpha=\alpha\left(p_{n}\right)=-v_{p_{n},+}^{\prime}(1)>0$ the unique maximal positive solution $u_{\alpha\left(p_{n}\right)}$ to (2.7) vanishes at some radius $\rho_{\alpha\left(p_{n}\right)}$ such that $1<\rho_{\alpha\left(p_{n}\right)}<+\infty$. Then, gluing $v_{p_{n},+}$ and $u_{\alpha\left(p_{n}\right)}$ as in the proof of Proposition 3.3 we obtain a radial sign-changing solution to Problem (3.1). Hence $p_{n} \in \mathcal{A}$ which is a contradiction since $p_{n}>\sup \mathcal{A}$. The proof is complete.

Proposition 3.5. For any integer $k \geq 2$ and any $p \in \mathcal{A}$ there exists a radial sign-changing solution $u_{p}$ to (3.1) with $k$ nodal regions.

Proof. Let $p \in \mathcal{A}$. We argue by induction on $k$. The basic step $k=2$ is obvious by the definition of $\mathcal{A}$ (see also Remark 3.1).

Assume that there exists $u_{p, k}$ radial sign-changing solution to (3.1) with $k$ nodal domains. We need to distinguish between two cases.

If $k$ is even, then $u_{p, k}<0$ in the last nodal region and thus by Hopf's Lemma we infer that $u_{p, k}^{\prime}(1)>0$. Then, since $p<\sup \mathcal{A}<p_{+}^{*}$, from [11. Theorem 1.1] we have that for any $\alpha>0$ the unique maximal positive solution $u_{\alpha}=u(\alpha, p, r)$ of the initial value problem (2.10) vanishes at some $\rho_{\alpha}<+\infty$. Hence, taking $\alpha=\alpha(p)=u_{p, k}^{\prime}(1)>0$, gluing $u_{p, k}, u_{\alpha(p)}$ as in the proof of Proposition 3.3 and rescaling, we obtain a radial solution $u_{p, k+1}$ of (3.1) with $k+1$ nodal regions and such that $u_{p, k+1}(0)>0$. This complete the proof of the inductive step when $k$ is even.

If $k$ is odd the previous argument works only for $p \leq p_{-}^{*}$ (see [11, Theorem 1.2]). For this reason we proceed in a different way. Let $u_{p, k}$ be a radial sign-changing solution to (3.1) with $k$ nodal regions. Since $k$ is odd then $u_{p, k}>0$ in the last nodal region and thus $-u_{p, k}^{\prime}(1)>0$. We claim that $-u_{p, k}^{\prime}(1)>\alpha_{-}^{*}(p)$, where $\alpha_{-}^{*}$ is the critical slope defined in (2.8). We first observe that if the claim is true then the maximal positive solution $u_{\alpha}=u(\alpha, p, r)$ of (2.7) with $\alpha=\alpha(p)=-u_{p, k}^{\prime}(1)$ vanishes at some $\rho_{\alpha(p)}<+\infty$, and thus we can construct $u_{p, k+1}$ satisfying the desired properties by gluing $u_{p, k}$ and $u_{\alpha(p)}$ in the same way as before.

To prove the claim, let $v_{p,+}$ be the only positive solution to (3.4) and consider its trajectory $\gamma_{1}(t)=\left(x_{1}(t), x_{1}^{\prime}(t)\right), t \in(-\infty, 0]$ in the phase-plane, where $x_{1}$ is the Emden-Fowler transform of $v_{p,+}$, obtained by setting $r=e^{t}$ and

$$
x_{1}(t):=e^{\frac{2}{p-1} t} v_{p,+}\left(e^{t}\right) .
$$

By construction $\gamma_{1}$ lies in the right-half plane and it is elementary to check that $\gamma_{1}(t) \rightarrow(0,0)$ as $t \rightarrow-\infty$, and $\gamma_{1}(0)=\left(0, v_{p,+}^{\prime}(1)\right)$, with $v_{p,+}^{\prime}(1)<0$. On the other hand, if we transform the restriction of $u_{p, k}$ to its last nodal component we obtain a trajectory $\gamma_{2}(t)=\left(x_{2}(t), x_{2}^{\prime}(t)\right)$, $t \in\left[\log \left(r_{k-1}\right), 0\right]$ lying in the right-half plane and such that $\gamma_{2}\left(\log \left(r_{k-1}\right)\right)=\left(0, r_{k-1}^{\frac{p+1}{p-1}} u_{p, k}^{\prime}\left(r_{k-1}\right)\right)$ 
with $u_{p, k}^{\prime}\left(r_{k-1}\right)>0$, while $\gamma_{2}(0)=\left(0, u_{p, k}^{\prime}(1)\right)$ and $u_{p, k}^{\prime}(1)<0$. Moreover, since $x_{1}, x_{2}$ satisfy the same autonomous ODE (see [11 for more details) then $\gamma_{1}$ and $\gamma_{2}$ cannot intersect and thus it follows that $0>v_{p,+}^{\prime}(1)>u_{p, k}^{\prime}(1)$.

Now, let us prove that $-v_{p,+}^{\prime}(1)>\alpha_{-}^{*}(p)$. Indeed, since $1<p<\sup \mathcal{A}$ and $p \in \mathcal{A}$ we know that there exists a radial sign-changing $u_{p, 2}$ solution to (3.1) with two nodal regions. Then, denoting by $r_{1} \in(0,1)$ the node of $u_{p, 2}$ and considering the usual scaling $\tilde{u}_{p, 2}(x):=r_{1}^{\frac{2}{p-1}} u_{p, 2}\left(r_{1} x\right)$, $x \in B_{1 / r_{1}}$, we infer that the restriction $\left.\tilde{u}_{p, 2}\right|_{B}$ coincides with $v_{p,+}$ (uniqueness of the positive radial solution) and the restriction $\left.\tilde{u}_{p, 2}^{-}\right|_{A_{1,1 / r_{1}}}$ coincides with the unique positive solution to (2.7) with $\alpha=-v_{p,+}^{\prime}(1)$. Therefore, since $\left.\tilde{u}_{p, 2}\right|_{A_{1,1 / r_{1}}}$ vanishes at $1 / r_{1}$ then by definition of $\alpha_{-}^{*}$ and by Theorem 2.2 we conclude that $-v_{p,+}^{\prime}(1)>\alpha_{-}^{*}(p)$.

Then we have proved that $\alpha_{-}^{*}(p)<-v_{p,+}^{\prime}(1)<-u_{p, k}^{\prime}(1)$. This concludes the proof.

Proof of Theorem 1.1. To prove i) we observe that the existence of radial sign-changing solutions to (1.1) for $\mathcal{F}=\mathcal{M}_{\lambda, \Lambda}^{-}$is a consequence of the existence of the positive solution of the same problem, combined with the Liouville type results obtained in [11. For this, let $p<p_{-}^{*}$ and let $v_{p}$ be the positive solution of

$$
\left\{\begin{array}{cl}
-\mathcal{M}_{\lambda, \Lambda}^{-}\left(D^{2} u\right)=|u|^{p-1} u & \text { in } B \\
u=0 & \text { on } \partial B \\
u(0)>0 &
\end{array}\right.
$$

By Hopf's Lemma it holds that $\left|v_{p}^{\prime}(1)\right|>0$. Let $w_{p}$ be the positive maximal solution of (2.10) with initial slope $w_{p}^{\prime}(1)=\left|v_{p}^{\prime}(1)\right|$. Since $p<p_{-}^{*}$ (which in particular implies that $p<p_{+}^{*}$ ) then in view of [11, Theorem 3.1], the function $w_{p}$ must vanish at some $\rho=\rho(p)>1$. Hence the function

$$
u_{p}(r)= \begin{cases}v_{p}(r) & \text { if } r \leq 1 \\ -w_{p}(r) & \text { if } r \in(1, \rho]\end{cases}
$$

defines a radial sign-changing solution, with two nodal regions, of

$$
-\mathcal{M}_{\lambda, \Lambda}^{-}\left(D^{2} u\right)=|u|^{p-1} u \quad \text { in } B_{\rho}
$$

and such that $u_{p}(0)>0$. By scaling, $\tilde{u}_{p}(r)=\rho^{\frac{2}{p-1}} u_{p}(\rho r)$ is a sign-changing solution of (3.6). This completes the proof of i) in the case of two nodal regions.

Let us point out that such gluing argument can be performed inductively, so providing the existence of sign-changing solutions for any number of nodal regions. The key point in this procedure is that $p$ is subcritical both for $\mathcal{M}_{\lambda, \Lambda}^{-}$and $\mathcal{M}_{\lambda, \Lambda}^{+}$, which implies that for any choice of the initial slope $\alpha>0$ the unique positive solution of (2.7) or (2.10) vanishes at some finite $\rho \in(1,+\infty)$ (see [11, Theorem 3.1]).

Let us prove ii). We define

$$
p_{+}^{* *}:=\sup \mathcal{A},
$$

where $\mathcal{A}$ is given by (3.2). Then ii) is a consequence of Proposition 3.2, Proposition 3.3 Proposition 3.4 and Proposition 3.5

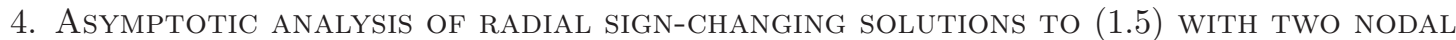 REGIONS}

In this section $u_{\varepsilon}$ will denote a radial sign-changing solution of (1.5) with two nodal regions. We set

$$
M_{0}=M_{0}(\varepsilon):=\left\|u_{\varepsilon}^{+}\right\|_{\infty}=u_{\varepsilon}^{+}(0),
$$


where $u_{\varepsilon}^{+}:=\max \left\{0, u_{\varepsilon}\right\}$ is the positive part of $u_{\varepsilon}$, and we denote by $r_{1}=r_{1}(\varepsilon) \in(0,1)$ the node of $u_{\varepsilon}$, i.e. the unique point $r_{1} \in(0,1)$ such that $u_{\varepsilon}\left(r_{1}\right)=0$. As in the previous section, $v_{p_{\varepsilon},-}$ stands for the only positive solution of (1.5). We begin with a preliminary result.

Proposition 4.1. The following statements hold:

i) $M_{0}>\left\|v_{p_{\varepsilon},-}\right\|_{\infty}$;

ii) $r_{1}=\left(\frac{\left\|v_{p_{\varepsilon},-}\right\|_{\infty}}{M_{0}}\right)^{\frac{p_{\varepsilon}-1}{2}}$;

iii) $\lim _{\varepsilon \rightarrow 0} M_{0}^{\frac{p_{\varepsilon}-1}{2}} r_{1}=+\infty$;

iv) $r_{1}^{\frac{p_{\varepsilon}+1}{p_{\varepsilon}-1}} u_{\varepsilon}^{\prime}\left(r_{1}\right)=\left(v_{p_{\varepsilon},-}\right)^{\prime}(1)$.

Proof. Consider the rescaled function $\tilde{u}_{\varepsilon}(x):=r_{1}^{\frac{2}{p_{\varepsilon}-1}} u_{\varepsilon}\left(r_{1} x\right), x \in B$. It is elementary to check that $\tilde{u}_{\varepsilon}$ is a positive radial solution to (1.5). Hence, by uniqueness, we infer that $\tilde{u}_{\varepsilon}=v_{p_{\varepsilon},-}$, and the result easily follows from Proposition 2.1.

Concerning the negative part of $u_{\varepsilon}$, namely $u_{\varepsilon}^{-}:=\max \left\{-u_{\varepsilon}, 0\right\}$, we adopt the following notations:

$$
M_{1}=M_{1}(\varepsilon):=\left\|u_{\varepsilon}^{-}\right\|_{\infty},
$$

$s_{1}=s_{1}(\varepsilon) \in\left(r_{1}, 1\right)$ is the point where the maximum $M_{1}$ is attained, i.e. $M_{1}=u_{\varepsilon}^{-}\left(s_{1}\right)$, and $t_{1} \in\left(s_{1}, 1\right)$ is the only radius such that

$$
\left(u_{\varepsilon}^{-}\right)^{\prime \prime}(r)<0 \quad \text { for } r \in\left(r_{1}, t_{1}\right), \quad\left(u_{\varepsilon}^{-}\right)^{\prime \prime}(r)>0 \quad \text { for } r \in\left(t_{1}, 1\right) .
$$

Remark 4.2. We point out that $M_{1}$ is bounded away from zero, in fact it is bounded from below by the principal eigenvalue $\lambda_{1}^{+}=\lambda_{1}^{+}\left(-\mathcal{M}_{\lambda, \Lambda}^{+} ; B\right)$. Indeed $u_{\varepsilon}^{-}$satisfies in the annulus $A_{r_{1}, 1}=\left\{x \in \mathbb{R}^{N} ; r_{1}<|x|<1\right\}$ the following

$$
\left\{\begin{array}{cl}
-\mathcal{M}_{\lambda, \Lambda}^{+}\left(D^{2} u_{\varepsilon}^{-}\right) \leq M_{1}^{p_{\varepsilon}-1} u_{\varepsilon}^{-} & \text {in } A_{r_{1}, 1} \\
u_{\varepsilon}^{-}=0 & \text { on } \partial A_{r_{1}, 1} .
\end{array}\right.
$$

Since the principal eigenvalue $\lambda_{1}^{+}$gives a threshold for the validity of the maximum principle (see [5]) and $u_{\varepsilon}^{-}>0$ in $A_{r_{1}, 1}$, then necessarily

$$
M_{1}^{p_{\varepsilon}-1} \geq \lambda_{1}^{+}\left(-\mathcal{M}_{\lambda, \Lambda}^{+} ; A_{r_{1}, 1}\right) \geq \lambda_{1}^{+}\left(-\mathcal{M}_{\lambda, \Lambda}^{+} ; B\right) .
$$

Proposition 4.3. The following statements hold:

i) $\lim _{\varepsilon \rightarrow 0} r_{1}=0$;

ii) $\lim _{\varepsilon \rightarrow 0} M_{1}^{\frac{p_{\varepsilon}-1}{2}} r_{1}=0$;

iii) $\lim _{\varepsilon \rightarrow 0} \frac{r_{1}}{s_{1}}=0$;

iv) $\lim _{\varepsilon \rightarrow 0}\left|u_{\varepsilon}^{\prime}\left(r_{1}\right)\right|=+\infty$.

Proof. Let us consider the energy-like functional

$$
H(r):=\frac{\left(u_{\varepsilon}^{\prime}(r)\right)^{2}}{2}+\frac{\left|u_{\varepsilon}(r)\right|^{p_{\varepsilon}+1}}{\lambda\left(p_{\varepsilon}+1\right)}, \quad r \in[0,1] .
$$

By a straightforward computation it holds that $H^{\prime} \leq 0$ in $\left[r_{1}, s_{1}\right]$ (actually it could be proved that $H^{\prime} \leq 0$ in the whole interval $\left.[0,1]\right)$. This in particular yields

$$
\frac{M_{1}^{p_{\varepsilon}+1}}{\lambda\left(p_{\varepsilon}+1\right)}=H\left(s_{1}\right) \leq H\left(r_{1}\right)=\frac{\left(u_{\varepsilon}^{\prime}\left(r_{1}\right)\right)^{2}}{2} .
$$


Using Proposition 4.1 riv), Proposition 2.1 ri,vi), (4.2) and (4.4) we then obtain

$$
\begin{aligned}
r_{1}^{2 \frac{p_{\varepsilon}+1}{p_{\varepsilon}-1}} & =\frac{\left(v_{p_{\varepsilon},-}^{\prime}(1)\right)^{2}}{\left(u_{\varepsilon}^{\prime}\left(r_{1}\right)\right)^{2}} \leq \frac{\lambda\left(p_{\varepsilon}+1\right)}{2} \frac{\left(v_{p_{\varepsilon},-}^{\prime}(1)\right)^{2}}{M_{1}^{p_{\varepsilon}+1}} \\
& \leq \frac{\lambda\left(p_{\varepsilon}+1\right)}{2} \frac{\left(v_{p_{\varepsilon},-}^{\prime}(1)\right)^{2}}{\lambda_{1}^{+}\left(-\mathcal{M}_{\lambda, \Lambda}^{+}, B\right)^{\frac{p_{\varepsilon}+1}{p_{\varepsilon}-1}}} \rightarrow 0 \quad \text { as } \varepsilon \rightarrow 0 .
\end{aligned}
$$

This proves i). Moreover, again by (4.4) and Proposition 4.1 tiv)

$$
r_{1} M_{1}^{\frac{p_{\varepsilon}-1}{2}} \leq\left(\frac{\lambda\left(p_{\varepsilon}+1\right)}{2}\right)^{\frac{p_{\varepsilon}-1}{2\left(p_{\varepsilon}+1\right)}} r_{1}\left|u_{\varepsilon}^{\prime}\left(r_{1}\right)\right|^{\frac{p_{\varepsilon}-1}{p_{\varepsilon}+1}}=\left(\frac{\lambda\left(p_{\varepsilon}+1\right)}{2}\right)^{\frac{p_{\varepsilon}-1}{2\left(p_{\varepsilon}+1\right)}}\left|v_{p_{\varepsilon},-}^{\prime}(1)\right|^{\frac{p_{\varepsilon}-1}{p_{\varepsilon}+1}}
$$

which proves ii).

In order to prove iii) and iv) let us consider the energy-like functionals

$$
H_{\gamma, \eta}(r):=r^{\tilde{N}_{-}}\left(\frac{\left(u_{\varepsilon}^{\prime}(r)\right)^{2}}{2}+\frac{\gamma}{p_{\varepsilon}+1}\left|u_{\varepsilon}(r)\right|^{p_{\varepsilon}+1}\right)+\eta r^{\tilde{N}_{-}-1} u_{\varepsilon}(r) u_{\varepsilon}^{\prime}(r),
$$

where $\gamma, \eta$ are real parameters to be chosen later. It is easy to check that for $r \in\left[r_{1}, s_{1}\right]$

$$
\begin{aligned}
H_{\gamma, \eta}^{\prime}(r)= & \left(\eta+1-\frac{\tilde{N}_{-}}{2}\right) r^{\tilde{N}_{-}-1}\left(u_{\varepsilon}^{\prime}(r)\right)^{2} \\
& +\left(\frac{\gamma \tilde{N}_{-}}{p_{\varepsilon}+1}-\frac{\eta}{\lambda}\right) r^{\tilde{N}_{-}-1}\left|u_{\varepsilon}(r)\right|^{p_{\varepsilon}+1} \\
& +\left(\gamma-\frac{1}{\lambda}\right) r^{\tilde{N}_{-}}\left|u_{\varepsilon}(r)\right|^{p_{\varepsilon}-1} u_{\varepsilon}(r) u_{\varepsilon}^{\prime}(r) .
\end{aligned}
$$

Choose

$$
\eta=\frac{\tilde{N}_{-}-2}{2}, \quad \gamma=\frac{\eta\left(p_{\varepsilon}+1\right)}{\lambda \tilde{N}_{-}}
$$

in (4.7). Since $p_{\varepsilon}>\frac{\tilde{N}_{-}+2}{\tilde{N}_{-}-2}$ for small $\varepsilon>0$, then $H_{\gamma, \eta}^{\prime} \geq 0$ in $\left[r_{1}, s_{1}\right]$. Hence $H_{\gamma, \eta}\left(r_{1}\right) \leq H_{\gamma, \eta}\left(s_{1}\right)$ which reads as

$$
r_{1}^{\tilde{N}_{-}}\left(u_{\varepsilon}^{\prime}\left(r_{1}\right)\right)^{2} \leq \frac{\tilde{N}_{-}-2}{\lambda \tilde{N}_{-}} s_{1}^{\tilde{N}_{-}} M_{1}^{p_{\varepsilon}+1} .
$$

By the convexity of $u_{\varepsilon}$ in $\left[r_{1}, s_{1}\right]$ we also have

$$
M_{1}^{2} \leq\left(u_{\varepsilon}^{\prime}\left(r_{1}\right)\right)^{2} s_{1}^{2} .
$$

Putting together (4.8)-(4.9) we get

$$
\left(\frac{r_{1}}{s_{1}}\right)^{\tilde{N}_{-}} \leq \frac{\tilde{N}_{-}-2}{\lambda \tilde{N}_{-}} M_{1}^{p_{\varepsilon}-1} s_{1}^{2}=\frac{\tilde{N}_{-}-2}{\lambda \tilde{N}_{-}} M_{1}^{p_{\varepsilon}-1} r_{1}^{2}\left(\frac{s_{1}}{r_{1}}\right)^{2} .
$$

Hence, by ii), iii) follows.

Now set in (4.6)-(4.7)

$$
\eta=\frac{\tilde{N}_{-}-2}{2}, \quad \gamma=\frac{1}{\lambda}
$$


With such a choice we easily check that $H_{\gamma, \eta}^{\prime} \leq 0$ in $\left[r_{1}, s_{1}\right]$. Then $H_{\gamma, \eta}\left(r_{1}\right) \geq H_{\gamma, \eta}\left(s_{1}\right)$ and using (4.2) we infer that

$$
\begin{aligned}
\left(u_{\varepsilon}^{\prime}\left(r_{1}\right)\right)^{2} & \geq \frac{2}{\lambda\left(p_{\varepsilon}+1\right)}\left(\frac{s_{1}}{r_{1}}\right)^{\tilde{N}_{-}} M_{1}^{p_{\varepsilon}+1} \\
& \geq \frac{2}{\lambda\left(p_{\varepsilon}+1\right)}\left(\frac{s_{1}}{r_{1}}\right)^{\tilde{N}_{-}}\left(\lambda_{1}^{+}\left(-\mathcal{M}_{\lambda, \Lambda}^{+}, B\right)\right)^{\frac{p_{\varepsilon}+1}{p_{\varepsilon}-1}} .
\end{aligned}
$$

This implies iv) because of iii).

In the following statements we will make use of the rescaled function defined by

$$
\hat{u}_{\varepsilon}^{-}(x):=\frac{1}{M_{1}} u_{\varepsilon}^{-}\left(\frac{x}{M_{1}^{\frac{p_{\varepsilon}-1}{2}}}\right) \quad x \in \hat{A}_{\varepsilon}
$$

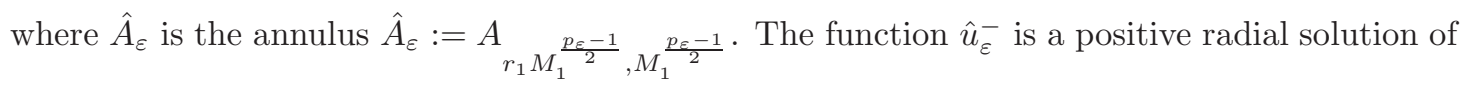

$$
\left\{\begin{aligned}
-\mathcal{M}_{\lambda, \Lambda}^{+}\left(D^{2} u\right) & =u^{p_{\varepsilon}} & & \text { in } \hat{A}_{\varepsilon}, \\
u & =0 & & \text { on } \partial \hat{A}_{\varepsilon} .
\end{aligned}\right.
$$

For convenience of notations we set

$$
\hat{r}_{1}=\hat{r}_{1}(\varepsilon):=r_{1} M_{1}^{\frac{p_{\varepsilon}-1}{2}}, \quad \hat{s}_{1}=\hat{s}_{1}(\varepsilon):=s_{1} M_{1}^{\frac{p_{\varepsilon}-1}{2}}, \quad \hat{t}_{1}=\hat{t}_{1}(\varepsilon):=t_{1} M_{1}^{\frac{p_{\varepsilon}-1}{2}} .
$$

The first result is about the asymptotic behavior of $\hat{s}_{1}$.

Proposition 4.4. $\lim _{\varepsilon \rightarrow 0} \hat{s}_{1}=0$.

Proof. We first prove that $\hat{s}_{1}$ is bounded from above. For this let us consider the energy function (4.3), which is monotone decreasing in $\left[r_{1}, s_{1}\right]$ (actually in $[0,1]$ ). Hence for any $r \in\left[r_{1}, s_{1}\right]$ we have $H(r) \geq H\left(s_{1}\right)$ and

$$
\left(u_{\varepsilon}^{-}\right)^{\prime}(r) \geq \sqrt{\frac{2}{\lambda\left(p_{\varepsilon}+1\right)}} \sqrt{\left(M_{1}^{p_{\varepsilon}+1}-\left(u_{\varepsilon}^{-}(r)\right)^{p_{\varepsilon}+1}\right)} .
$$

Integrating in $\left[r_{1}, s_{1}\right]$ we infer that

$$
\int_{r_{1}}^{s_{1}} \frac{\left(u_{\varepsilon}^{-}\right)^{\prime}(r)}{\sqrt{\left(M_{1}^{p_{\varepsilon}+1}-u_{\varepsilon}^{-}(r)^{p_{\varepsilon}+1}\right)}} d r \geq \sqrt{\frac{2}{\lambda\left(p_{\varepsilon}+1\right)}}\left(s_{1}-r_{1}\right) .
$$

With the change of variable $t=\frac{u_{\varepsilon}^{-}}{M_{1}}$ we obtain

$$
\int_{r_{1}}^{s_{1}} \frac{\left(u_{\varepsilon}^{-}\right)^{\prime}(r)}{\sqrt{\left(M_{1}^{p_{\varepsilon}+1}-u_{\varepsilon}^{-}(r)^{p_{\varepsilon}+1}\right)}} d r=\frac{1}{M_{1}^{\frac{p_{\varepsilon}-1}{2}}} \int_{0}^{1} \frac{1}{\sqrt{1-t^{p_{\varepsilon}+1}}} d t .
$$

Then from (4.14) we deduce that

$$
\hat{s}_{1}-\hat{r}_{1} \leq \sqrt{\frac{\lambda\left(p_{\varepsilon}+1\right)}{2}} \int_{0}^{1} \frac{d t}{\sqrt{1-t^{p_{\varepsilon}+1}}} .
$$

Sending $\varepsilon \rightarrow 0$ and using Proposition 4.3 tii)

$$
\limsup _{\varepsilon \rightarrow 0} \hat{s}_{1} \leq \sqrt{\frac{\lambda\left(p_{-}^{*}+1\right)}{2}} \int_{0}^{1} \frac{d t}{\sqrt{1-t^{p_{-}^{*}+1}}} \leq \frac{\pi}{2} \sqrt{\frac{\lambda N}{N-2}},
$$


which proves the claim.

From (2.4) it is easy to check that

$$
\left(r^{\tilde{N}_{-}-1}\left(u_{\varepsilon}^{-}\right)^{\prime}\right)^{\prime}+\frac{1}{\lambda} r^{\tilde{N}_{-}-1} u_{\varepsilon}^{-}(r)^{p_{\varepsilon}}=0 \quad \text { in }\left[r_{1}, s_{1}\right] .
$$

Integrating from $r_{1}$ to $r \in\left[r_{1}, s_{1}\right]$ we obtain

$$
-r^{\tilde{N}_{-}-1}\left(u_{\varepsilon}^{-}\right)^{\prime}(r)+r_{1}^{\tilde{N}_{-}-1}\left(u_{\varepsilon}^{-}\right)^{\prime}\left(r_{1}\right)=\frac{1}{\lambda} \int_{r_{1}}^{r} s^{\tilde{N}_{-}-1} u_{\varepsilon}^{-}(s)^{p_{\varepsilon}} d s \leq \frac{M_{1}^{p_{\varepsilon}}}{\lambda \tilde{N}_{-}} r^{\tilde{N}_{-}}
$$

and then

$$
-\left(u_{\varepsilon}^{-}\right)^{\prime}(r) \leq \frac{M_{1}^{p_{\varepsilon}}}{\lambda \tilde{N}_{-}} r-r_{1}^{\tilde{N}_{-}-1}\left(u_{\varepsilon}^{-}\right)^{\prime}\left(r_{1}\right) r^{1-\tilde{N}_{-}} \quad \text { in }\left[r_{1}, s_{1}\right] .
$$

Integrating the above inequality in $\left[r_{1}, s_{1}\right]$, we infer that

$$
\frac{r_{1}\left(u_{\varepsilon}^{-}\right)^{\prime}\left(r_{1}\right)}{\tilde{N}_{-}-2}\left[1-\left(\frac{r_{1}}{s_{1}}\right)^{\tilde{N}_{-}-2}\right] \leq M_{1}\left(1+\frac{\hat{s}_{1}^{2}}{2 \lambda \tilde{N}_{-}}\right) \text {. }
$$

Since $\hat{s}_{1}$ is bounded from above and $\lim _{\varepsilon \rightarrow 0} \frac{r_{1}}{s_{1}} \rightarrow 0$, in view of Proposition 4.3 -iii), then

$$
r_{1}\left(u_{\varepsilon}^{-}\right)^{\prime}\left(\hat{r}_{1}\right) \leq C M_{1}
$$

for some positive constant $C$. Moreover $r_{1}\left(u_{\varepsilon}^{-}\right)^{\prime}\left(r_{1}\right) \geq 0$. Hence using (4.10)

$$
\begin{aligned}
C^{2} & \geq\left(r_{1} \frac{\left(u_{\varepsilon}^{-}\right)^{\prime}\left(r_{1}\right)}{M_{1}}\right)^{2} \\
& \geq \frac{2}{\lambda\left(p_{\varepsilon}+1\right)}\left(\frac{s_{1}}{r_{1}}\right)^{\tilde{N}_{-}-2} \hat{s}_{1}^{2} .
\end{aligned}
$$

The conclusion follows by Proposition 4.3 iii).

From the previous result we immediately deduce that $\hat{r}_{1} \rightarrow 0$, as $\varepsilon \rightarrow 0$. Moreover we have

Corollary 4.5. $\lim _{\varepsilon \rightarrow 0} s_{1}=0$.

Proof. Use (4.2) and Proposition 4.4

Next we study the asymptotic behavior of $\hat{t}_{1}$ and show that it cannot converge to zero. We begin with a stronger result.

Proposition 4.6. $\liminf _{\varepsilon \rightarrow 0} t_{1}\left(u_{\varepsilon}^{-}\left(t_{1}\right)\right)^{\frac{p_{\varepsilon}-1}{2}} \geq \sqrt{\frac{2 \lambda(N-1)}{p_{-}^{*}+1}}$.

Proof. Let us consider the energy-like functional

$$
H(r):=r^{N}\left(\frac{\left[\left(u_{\varepsilon}^{-}\right)^{\prime}(r)\right]^{2}}{2}+\frac{1}{\lambda\left(p_{\varepsilon}+1\right)}\left(u_{\varepsilon}^{-}(r)\right)^{p_{\varepsilon}+1}\right)+\frac{N}{p_{\varepsilon}+1} r^{N-1} u_{\varepsilon}^{-}(r)\left(u_{\varepsilon}^{-}\right)^{\prime}(r), \quad r \in\left[s_{1}, t_{1}\right] .
$$

By (2.5), a direct computation shows that $H^{\prime}(r) \geq 0$. Hence $H\left(s_{1}\right) \leq H\left(t_{1}\right)$ and thus

$$
s_{1}^{N} \frac{M_{1}^{p_{\varepsilon}+1}}{\lambda\left(p_{\varepsilon}+1\right)} \leq t_{1}^{N}\left(\frac{\left[\left(u_{\varepsilon}^{-}\right)^{\prime}\left(t_{1}\right)\right]^{2}}{2}+\frac{1}{\lambda\left(p_{\varepsilon}+1\right)}\left(u_{\varepsilon}^{-}\left(t_{1}\right)\right)^{p_{\varepsilon}+1}\right)+\frac{N}{p_{\varepsilon}+1} t_{1}^{N-1} u_{\varepsilon}^{-}\left(t_{1}\right)\left(u_{\varepsilon}^{-}\right)^{\prime}\left(t_{1}\right) .
$$

Exploiting the equation

$$
(N-1) \frac{\left(u_{\varepsilon}^{-}\right)^{\prime}\left(t_{1}\right)}{t_{1}}=-\frac{1}{\lambda}\left(u_{\varepsilon}^{-}\left(t_{1}\right)\right)^{p_{\varepsilon}},
$$


we have

$$
s_{1}^{N} \frac{M_{1}^{p_{\varepsilon}+1}}{\lambda\left(p_{\varepsilon}+1\right)} \leq \frac{1}{\lambda(N-1)} t_{1}^{N}\left(u_{\varepsilon}^{-}\left(t_{1}\right)\right)^{p_{\varepsilon}+1}\left(\frac{1}{2 \lambda(N-1)} t_{1}^{2}\left(u_{\varepsilon}^{-}\left(t_{1}\right)\right)^{p_{\varepsilon}-1}-\frac{1}{p_{\varepsilon}+1}\right) .
$$

This implies that

$$
t_{1}^{2}\left(u_{\varepsilon}^{-}\left(t_{1}\right)\right)^{p_{\varepsilon}-1} \geq \frac{2 \lambda(N-1)}{p_{\varepsilon}+1}
$$

and the conclusion follows passing to the limit as $\varepsilon \rightarrow 0$.

As an immediate consequence of the previous proposition we get

Corollary 4.7. $\liminf _{\varepsilon \rightarrow 0} \hat{t}_{1} \geq \sqrt{\frac{2 \lambda(N-1)}{p_{-}^{*}+1}}$.

Proof. It suffices to observe that $\hat{t}_{1}=t_{1} M_{1}^{\frac{p_{\varepsilon}-1}{2}} \geq t_{1}\left(u_{\varepsilon}^{-}\left(t_{1}\right)\right)^{\frac{p_{\varepsilon}-1}{2}}$ so that the assertion follows by Proposition 4.6

Coming back to the study of $u_{\varepsilon}^{-}$, from the previous results we obtain

Proposition 4.8. $\limsup _{\varepsilon \rightarrow 0} M_{1}<+\infty$.

Proof. Arguing by contradiction let us assume that along some subsequence, still denoted by $\varepsilon$, $M_{1} \rightarrow \infty$. Consider the rescaled function $\hat{u}_{\varepsilon}^{-}$defined in (4.11). By construction $\hat{u}_{\varepsilon}^{-}$satisfies

$$
\hat{u}_{\varepsilon}^{-}\left(\hat{s}_{1}\right)=1, \quad 0 \leq \hat{u}_{\varepsilon}^{-} \leq 1 .
$$

The limit of the domains $\hat{A}_{\varepsilon}$ is $\mathbb{R}^{N} \backslash\{0\}$ and, since $\left(\hat{u}_{\varepsilon}^{-}\right)_{\varepsilon}$ is uniformly bounded and solves (4.12), by regularity estimates, we have that $\hat{u}_{\varepsilon}^{-} \rightarrow \hat{u}$ in $C_{\mathrm{loc}}^{2}\left(\mathbb{R}^{N} \backslash\{0\}\right)$, for some radially symmetric function $\hat{u}, 0 \leq \hat{u} \leq 1$, which solves

$$
-\mathcal{M}_{\lambda, \Lambda}^{+}\left(D^{2} u\right)=u^{p_{-}^{*}} \quad \text { in } \mathbb{R}^{N} \backslash\{0\} .
$$

Now we want to show that $\hat{u}$ can be extended to a $C^{2}$ solution of 4.17) in the whole $\mathbb{R}^{N}$ with $\hat{u}(0)=1$ and $\hat{u}^{\prime}(0)=0$. In the interval $\left(\hat{s}_{1}, \hat{t}_{1}\right)$ we have that $\left(\hat{u}_{\varepsilon}^{-}\right)^{\prime} \leq 0$ and $\left(\hat{u}_{\varepsilon}^{-}\right)^{\prime \prime} \leq 0$. Therefore the equation satisfied by $\hat{u}_{\varepsilon}^{-}$is

$$
-\left(\hat{u}_{\varepsilon}^{-}\right)^{\prime \prime}(r)-\frac{N-1}{r}\left(\hat{u}_{\varepsilon}^{-}\right)^{\prime}(r)=\frac{\left(\hat{u}_{\varepsilon}^{-}\right)(r)^{p_{\varepsilon}}}{\lambda} \quad r \in\left(\hat{s}_{1}, \hat{t}_{1}\right)
$$

that we can write as:

$$
\left(r^{N-1}\left(\hat{u}_{\varepsilon}^{-}\right)^{\prime}\right)^{\prime}=-\frac{1}{\lambda} r^{N-1}\left(\hat{u}_{\varepsilon}^{-}\right)(r)^{p_{\varepsilon}} \geq-\frac{1}{\lambda} r^{N-1} .
$$

Integrating between $\hat{s}_{1}$ and $r \in\left(\hat{s}_{1}, \hat{t}_{1}\right)$ we get

$$
r^{N-1}\left(\hat{u}_{\varepsilon}^{-}\right)^{\prime}(r)-\hat{s}_{1}^{N-1}\left(\hat{u}_{\varepsilon}^{-}\right)^{\prime}\left(\hat{s}_{1}\right) \geq-\frac{1}{\lambda N}\left(r^{N}-\hat{s}_{1}^{N}\right)
$$

and, since $\left(\hat{u}_{\varepsilon}^{-}\right)^{\prime}\left(\hat{s}_{1}\right)=0$ and $\hat{s}_{1}>0$, we obtain

$$
\left(\hat{u}_{\varepsilon}^{-}\right)^{\prime}(r) \geq-\frac{1}{\lambda N} r \quad \text { for } r \in\left(\hat{s}_{1}, \hat{t}_{1}\right)
$$

Integrating again between $\hat{s}_{1}$ and $r \in\left(\hat{s}_{1}, \hat{t}_{1}\right)$, taking into account that $\hat{u}_{\varepsilon}^{-}\left(\hat{s}_{1}\right)=1$, we have

$$
\hat{u}_{\varepsilon}^{-}(r) \geq 1-\frac{1}{2 \lambda N} r^{2} \quad \text { for } r \in\left(\hat{s}_{1}, \hat{t}_{1}\right) .
$$


Passing to the limit as $\varepsilon \rightarrow 0$, taking into account Proposition 4.4 and Corollary 4.7 , we infer that

$$
\hat{u}(r) \geq 1-\frac{1}{2 \lambda N} r^{2} \quad \text { for } r \in\left(0, \sqrt{\frac{2 \lambda(N-1)}{p_{-}^{*}+1}}\right) .
$$

From this, since $\hat{u} \leq 1$, we deduce that

$$
\lim _{r \rightarrow 0} \hat{u}(r)=1 .
$$

Hence $\hat{u}$ can be extended by continuity at the origin, by setting $\hat{u}(0):=1$. Next we show that also $\hat{u}^{\prime}$ can be extended by continuity in 0 . From (4.19) we have

$$
\left|\left(\hat{u}_{\varepsilon}^{-}\right)^{\prime}(r)\right| \leq \frac{r}{\lambda N} \quad \text { for any } r \in\left(\hat{s}_{1}, \hat{t}_{1}\right)
$$

and passing to the limit as $\varepsilon \rightarrow 0$, we get

$$
\left|\hat{u}^{\prime}(r)\right| \leq \frac{r}{\lambda N} \quad \text { for } r \in\left(0, \sqrt{\frac{2 \lambda(N-1)}{p_{-}^{*}+1}}\right)
$$

which gives $\lim _{r \rightarrow 0} \hat{u}^{\prime}(r)=0$. Hence $\hat{u}$ is a positive radial solution of (4.17) that extends to a $C^{1}$ function near the origin. This implies that $\hat{u}$ is a (positive) $C^{2}$ radial solution of

$$
-\mathcal{M}_{\lambda, \Lambda}^{+}\left(D^{2} u\right)=u^{p_{-}^{*}} \text { in } \mathbb{R}^{N} .
$$

Indeed, since $\hat{u}$ verifies $\left(r^{N-1} \hat{u}^{\prime}(r)\right)^{\prime}=-\frac{1}{\lambda} r^{N-1}(\hat{u}(r))^{p_{-}^{*}}$ for $r \in\left(0, \sqrt{\frac{2 \lambda(N-1)}{p_{-}^{*}+1}}\right)$, then, fixing $0<\delta<r$ and integrating between $\delta$ and $r$, we get that

$$
r^{N-1} \hat{u}^{\prime}(r)-\delta^{N-1} \hat{u}^{\prime}(\delta)=-\frac{1}{\lambda} \int_{\delta}^{r} s^{N-1}(\hat{u}(s))^{p_{-}^{*}} d s .
$$

Passing to the limit as $\delta \rightarrow 0$, by (4.22), we obtain

$$
\frac{\hat{u}^{\prime}(r)}{r}=-\frac{1}{\lambda r^{N}} \int_{0}^{r} s^{N-1}(\hat{u}(s))^{p_{-}^{*}} d s .
$$

By de L'Hôpital's rule, the right-hand side of (4.24) has a finite limit as $r \rightarrow 0$ and thus the same holds for the left-hand side, which readily implies that $\hat{u}$ extends to a $C^{2}$ radial solution of (4.23).

At the end, since $p_{-}^{*}<p_{+}^{*}$, then by 9 , Theorem 1.1] we know that (4.23) has only the trivial solution, which contradicts the positivity of $\hat{u}$.

Summing up, we have all the ingredients to prove the following

Theorem 4.9. Let $u_{\varepsilon}$ be a radial sign-changing solution to (1.5) with two nodal regions. Then, up to a subsequence, as $\varepsilon \rightarrow 0^{+}$we have that $u_{\varepsilon} \rightarrow \bar{u}$ in $C_{l o c}^{2}(\bar{B} \backslash\{0\})$, where $\bar{u}$ is the unique negative solution of (1.6)

Proof. Let us consider the restriction of $u_{\varepsilon}^{-}$to the annulus $A_{r_{1}, 1}$. From Proposition 4.8 we have that $\left.u_{\varepsilon}^{-}\right|_{A_{r_{1}, 1}}$ is uniformly bounded and from Proposition 4.3, i) we have $r_{1} \rightarrow 0$. Hence, by standard regularity theory, up to a subsequence as $\varepsilon \rightarrow 0$, we get that $u_{\varepsilon}^{-} \rightarrow \bar{u}^{-}$in $C_{l o c}^{2}(\bar{B} \backslash\{0\})$, where $\bar{u}^{-}$is a non-negative radial solution of

$$
\left\{\begin{array}{cl}
-\mathcal{M}_{\lambda, \Lambda}^{+}\left(D^{2} u\right)=|u|^{p_{-}^{*}-1} u & \text { in } B \backslash\{0\}, \\
u=0 & \text { on } \partial B .
\end{array}\right.
$$

We claim that $\bar{u}^{-}$can be extended to a smooth positive solution of (4.25) in the whole ball. For this, taking into account that $s_{1} \rightarrow 0$ by Corollary $4.5, u_{\varepsilon}^{-}\left(s_{1}\right)=M_{1} \rightarrow \bar{M}_{1} \in(0,+\infty)$ in view 
of Proposition 4.8 and (4.2), $t_{1} \rightarrow \bar{t}_{1} \in(0,1)$, as it follows by combining Proposition 4.8 and Proposition 4.6 (the case $\bar{t}_{1}=1$ being excluded by Hopf lemma), then repeating the proofs of (4.21), (4.22), with $\hat{u}_{\varepsilon}^{-}$replaced by $u_{\varepsilon}^{-}$, we get that

$$
\lim _{r \rightarrow 0} \bar{u}^{-}(r)=\bar{M}_{1}, \quad \lim _{r \rightarrow 0}\left(\bar{u}^{-}\right)^{\prime}(r)=0 .
$$

Hence $\bar{u}^{-}$extends to a $C^{1}$ radial function near the origin and we easily conclude as in the proof of Proposition 4.8 .

\section{Asymptotic analysis of Radial Sign-Changing SOlutions to (1.5) With three NODAL REGIONS}

To prove Theorem 1.2 we could argue by induction starting from $k=2$, nevertheless, for the reader's convenience, we detail the case $k=3$.

Let $u_{\varepsilon}$ be a sign-changing solution of (1.5) with three nodal regions, let $r_{i}=r_{i}(\varepsilon), i=1,2$, be the nodal radii, let $s_{i}=s_{i}(\varepsilon), i=1,2$, be the maximum points of $\left|u_{\varepsilon}\right|$ in the second and third nodal region, and denote by $M_{i}=M_{i}(\varepsilon), i=0,1,2$ the maximum values of $\left|u_{\varepsilon}\right|$ in each nodal region.

The following lemma is a trivial consequence of the results obtained in the previous section.

Lemma 5.1. As $\varepsilon \rightarrow 0^{+}$, we have: $r_{1} \rightarrow 0, s_{1} \rightarrow 0, \frac{r_{1}}{r_{2}} \rightarrow 0, M_{0} \rightarrow+\infty$ and

$$
0<\liminf r_{2}^{\frac{2}{p_{\varepsilon}-1}} M_{1} \leq \limsup r_{2}^{\frac{2}{p_{\varepsilon}-1}} M_{1}<+\infty .
$$

Proof. Let us consider the rescaled function

$$
\tilde{u}_{\varepsilon, 2}(x)=r_{2}^{\frac{2}{p_{-}-1}} u_{\varepsilon}\left(r_{2} x\right), \quad x \in B_{\frac{1}{r_{2}}} .
$$

By construction the restriction of $\tilde{u}_{\varepsilon, 2}$ to $B$ is a sign-changing solution to (1.5) with exactly two nodal regions, and thus we can apply the results of Sect. 4. In particular, denoting by $\tilde{r}_{1} \in(0,1)$ the nodal radius of $\tilde{u}_{\varepsilon, 2}$, by $\tilde{s}_{1}$ the maximum point of $\left|\tilde{u}_{\varepsilon, 2}^{-}\right|$, and setting $\tilde{M}_{0}:=\tilde{u}_{\varepsilon, 2}(0)$, $\tilde{M}_{1}:=\left|\tilde{u}_{\varepsilon, 2}\left(\tilde{s}_{1}\right)\right|$, as $\varepsilon \rightarrow 0^{+}$we have:

a) $\tilde{r}_{1}=\frac{r_{1}}{r_{2}} \rightarrow 0$

b) $\tilde{s}_{1}=\frac{s_{1}}{r_{2}} \rightarrow 0$

c) $\tilde{M}_{0}=r_{2}^{\frac{2}{p_{\varepsilon}-1}} M_{0} \rightarrow+\infty$,

d) $0<\liminf r_{2}^{\frac{2}{p_{\varepsilon}-1}} M_{1} \leq \limsup r_{2}^{\frac{2}{p_{\varepsilon}-1}} M_{1}<+\infty$.

Next we study the asymptotic behavior of the function $\tilde{u}_{\varepsilon, 2}$ defined in (5.1) in its third nodal region, which is the annulus $A_{1, \frac{1}{r_{2}}}$. To this end we set $\tilde{s}_{2}:=\frac{s_{2}}{r_{2}}, \tilde{M}_{2}:=r_{2}^{\frac{2}{p_{\varepsilon}-1}} M_{2}$, which are, respectively, the maximum point and the maximum value of $\tilde{u}_{\varepsilon, 2}$ achieved in $A_{1, \frac{1}{r_{2}}}$.

Proposition 5.2. $\limsup _{\varepsilon \rightarrow 0} \tilde{M}_{2}<+\infty$.

Proof. Let us consider the energy-like functionals $H_{\lambda}:\left[s_{1}, t_{1}\right] \rightarrow \mathbb{R}, H_{\Lambda}:\left[t_{1}, s_{2}\right] \rightarrow \mathbb{R}$ defined by

$$
\begin{aligned}
& H_{\lambda}(r):=\frac{\left(u_{\varepsilon}^{\prime}(r)\right)^{2}}{2}+\frac{\left|u_{\varepsilon}(r)\right|^{p_{\varepsilon}+1}}{\lambda\left(p_{\varepsilon}+1\right)}, \\
& H_{\Lambda}(r):=\frac{\left(u_{\varepsilon}^{\prime}(r)\right)^{2}}{2}+\frac{\left|u_{\varepsilon}(r)\right|^{p_{\varepsilon}+1}}{\Lambda\left(p_{\varepsilon}+1\right)},
\end{aligned}
$$


where $t_{1}$ is the only point contained in the interval $\left(r_{1}, r_{2}\right)$ such that $u_{\varepsilon}^{\prime \prime}\left(t_{1}\right)=0$. Exploiting the ODE in (2.7), taking into account that $u_{\varepsilon}^{\prime \prime} \geq 0, u_{\varepsilon}^{\prime} \geq 0$ in $\left[s_{1}, t_{1}\right]$ and $u_{\varepsilon}^{\prime \prime} \leq 0, u_{\varepsilon}^{\prime} \geq 0$ in $\left[t_{1}, s_{2}\right]$, we easily check that $H_{\lambda}$ and $H_{\Lambda}$ are decreasing. Hence, since $\lambda \leq \Lambda$, we infer that

$$
H_{\lambda}\left(s_{1}\right) \geq H_{\lambda}\left(t_{1}\right) \geq H_{\Lambda}\left(t_{1}\right) \geq H_{\Lambda}\left(s_{2}\right),
$$

which gives

From this we get that

$$
\frac{M_{1}^{p_{\varepsilon}+1}}{\lambda\left(p_{\varepsilon}+1\right)} \geq \frac{M_{2}^{p_{\varepsilon}+1}}{\Lambda\left(p_{\varepsilon}+1\right)}
$$

and using Lemma 5.1 we conclude.

$$
r_{2}^{\frac{2}{p \varepsilon-1}} M_{1} \geq\left(\frac{\lambda}{\Lambda}\right)^{\frac{1}{p_{\varepsilon}+1}} r_{2}^{\frac{2}{p \varepsilon-1}} M_{2}
$$

Lemma 5.3. $\liminf _{\varepsilon \rightarrow 0} r_{2}>0$.

Proof. Assume by contradiction that there exists a sequence $\varepsilon \rightarrow 0^{+}$such that $r_{2} \rightarrow 0$. Consider the restriction to $A_{\frac{r_{1}}{r_{2}}, \frac{1}{r_{2}}}$ of the rescaled function $\tilde{u}_{\varepsilon, 2}$ defined in (5.1). Since $r_{2} \rightarrow 0$ and thanks to Lemma 5.1 the limit domain of $A_{\frac{r_{1}}{r_{2}}, \frac{1}{r_{2}}}$ is $\mathbb{R}^{N} \backslash\{0\}$. Thanks to Lemma 5.1. Proposition 5.2 and elliptic regularity theory we infer that, up to a further subsequence, $\tilde{u}_{\varepsilon, 2} \rightarrow \tilde{u}$ in $C_{l o c}^{2}\left(\mathbb{R}^{N} \backslash\{0\}\right)$, for some radially symmetric function $\tilde{u}$ satisfying

$$
-\mathcal{M}_{\lambda, \Lambda}^{-}\left(D^{2} u\right)=|u|^{p_{-}^{*}-1} u \text { in } \mathbb{R}^{N} .
$$

Moreover, in view of Theorem 4.9 it holds that $\tilde{u}<0$ in $B$. Hence, since $\tilde{u}=0$ on $\partial B$, by Hopf's Lemma we get that $\tilde{u}^{\prime}(1)>0$. Therefore, for $r>1$ the function $\tilde{u}=\tilde{u}(r)$ is a solution (defined and positive in the whole $(1,+\infty)$ ) to (2.7) with $p=p_{-}^{*}, \alpha=\tilde{u}^{\prime}(1)$, but this contradicts [11, Theorem 1.1].

Corollary 5.4. $\liminf _{\varepsilon \rightarrow 0} \tilde{M}_{2}>0$.

Proof. Arguing as in Remark 4.2 we have $\tilde{M}_{2}^{p_{\varepsilon}-1} \geq \lambda_{1}^{+}\left(-\mathcal{M}_{\lambda, \Lambda}^{-} ; A_{1,1 / r_{2}}\right)$ and the conclusion follows from Lemma 5.3 .

Finally, summing up, we can describe the asymptotic behavior of $u_{\varepsilon}$.

Theorem 5.5. Let $u_{\varepsilon}$ be a radial sign-changing solution to (1.5) with three nodal regions. Then, up to a subsequence, as $\varepsilon \rightarrow 0^{+}$we have that $u_{\varepsilon} \rightarrow \bar{u}$ in $C_{l o c}^{2}(\bar{B} \backslash\{0\})$, where $\bar{u}$ is a radial sign-changing solution of (1.6) having two nodal regions.

Proof. We first observe that, as a consequence of Lemma 5.1 and Lemma 5.3, we have that $M_{1}$ is uniformly bounded, and bounded away from zero. The same holds for $M_{2}$ in view of (5.2) and Corollary 5.4. Moreover from Lemma 5.1 we know that $r_{1} \rightarrow 0$. Hence, the restriction of $u_{\varepsilon}$ to $A_{r_{1}, 1}$ is uniformly bounded and by standard regularity theory, up to a subsequence, $u_{\varepsilon} \rightarrow \bar{u}$ in $C_{l o c}^{2}(\bar{B} \backslash\{0\})$, for some radially symmetric function $\bar{u}$ satisfying (4.25).

We claim that $\bar{u}$ is sign-changing with exactly two nodal regions. To prove this we first notice that since $M_{2}$ is uniformly bounded we have $r_{2} \not \rightarrow 1$, otherwise $\lambda_{1}\left(-\mathcal{M}_{\lambda, \Lambda}^{-} ; A_{r_{2}, 1}\right) \rightarrow$ $+\infty$ and from the inequality $M_{2}^{p_{\varepsilon}-1} \geq \lambda_{1}\left(-\mathcal{M}_{\lambda, \Lambda}^{-} ; A_{r_{2}, 1}\right)$ (see Remark 4.2) we would obtain a contradiction. Hence, from this and Lemma 5.3 we infer that $r_{2} \rightarrow \bar{r}_{2} \in(0,1)$.

Now, since the restriction to the unit ball of $\tilde{u}_{\varepsilon, 2}$ defined in (5.1) is a sign-changing solution of (1.5) with two nodal regions, then from Theorem 4.9 we get that $\tilde{u}_{\varepsilon, 2}$ converges in $C_{l o c}^{2}(\bar{B} \backslash\{0\})$ to the unique negative radial solution $\tilde{u}$ of (1.6). Moreover, since $r_{2} \rightarrow \bar{r}_{2} \in(0,1)$ we deduce 
that $\bar{u}(x)=\bar{r}_{2}^{-\frac{2}{p_{-}^{*}-1}} \tilde{u}\left(\bar{r}_{2}^{-1} x\right)$ for $x \in B_{\bar{r}_{2}} \backslash\{0\}$. Therefore, $\bar{u}$ extends to a smooth function near the origin which is a radial solution of (1.6) and such that $\bar{u}<0$ in $B_{\bar{r}_{2}}$ and $\bar{u}\left(\bar{r}_{2}\right)=0$. Hence $\bar{u}^{\prime}\left(\bar{r}_{2}\right)>0$ and thus we easily deduce that $\bar{u}>0$ in $A_{\bar{r}_{2}, 1}$. Moreover we have $s_{2} \rightarrow \bar{s}_{2}$, for some $\bar{s}_{2}$ such that $\bar{r}_{2}<\bar{s}_{2}<1$. In fact, $s_{2} \rightarrow \bar{r}_{2}$ cannot happen because $\bar{u}^{\prime}\left(\bar{r}_{2}\right)>0$, while $s_{2} \not \rightarrow 1$ because $\bar{u}>0$ in $A_{\bar{r}_{2}, 1}, \bar{u}(1)=0$ and thus $\bar{u}^{\prime}(1)<0$.

\section{Asymptotic analysis of Radial Sign-ChAnging SOlutions to (1.5) With $k$ NOdAL REGIONS}

In this section we prove Theorem 1.2

Proof of Theorem 1.2. The case $k=2$ has been proved in Theorem 4.9. For $k \geq 3$ we argue by induction on $k$. The case $k=3$ is given by Theorem 5.5. Then, assuming the assertion true for a solution with $k$ nodal domains, let $u_{\varepsilon}$ be a radial sign-changing solution of (1.5) with $(k+1)$ nodal regions. Consider the rescaled function

$$
\tilde{u}_{\varepsilon, k}(x)=r_{k}^{\frac{2}{p_{\varepsilon}-1}} u_{\varepsilon}\left(r_{k} x\right), \quad x \in B_{\frac{1}{r_{k}}} .
$$

Denoting with $\tilde{r}_{i}, \tilde{s}_{i}, \tilde{M}_{i}$ the corresponding quantities for $\tilde{u}_{\varepsilon, k}$, by construction, we have $\tilde{M}_{i}=$ $r_{k}^{\frac{2}{p_{\varepsilon}-1}} M_{i}, \tilde{s}_{i}=\frac{s_{i}}{r_{k}}$ for $i=0, \ldots, k$ and $\tilde{r}_{i}=\frac{r_{i}}{r_{k}}$, for $i=1, \ldots, k$.

Now, since the restriction of $\tilde{u}_{\varepsilon, k}$ to the unit ball is a solution to (1.5) with $k$ nodal regions then, by the inductive hypothesis, up to a subsequence, as $\varepsilon \rightarrow 0^{+}$we get that $\tilde{M}_{0} \rightarrow+\infty, \tilde{r}_{1} \rightarrow 0$, $\tilde{s}_{1} \rightarrow 0$ and $\tilde{r}_{i} \rightarrow \overline{\tilde{r}_{i}}, \tilde{s}_{i} \rightarrow \overline{\tilde{s}_{i}}$, for $i=2, \ldots, k-1$, where $0<\overline{\tilde{r}_{2}}<\overline{\tilde{s}_{2}}<\ldots<\overline{\tilde{r}_{k-1}}<\overline{\tilde{s}_{k-1}}<1$ and $\tilde{M}_{i} \rightarrow \tilde{M}_{i}$, for some positive numbers $\overline{\tilde{M}_{i}}$, for $i=1, \ldots, k-1$. Moreover $\tilde{u}_{\varepsilon, k} \rightarrow \overline{\tilde{u}_{k}}$, in $C_{l o c}^{2}(\bar{B} \backslash\{0\})$, where $\overline{\tilde{u}_{k}}$ is a radial sign-changing solution to (1.6) with $k-1$ nodal regions.

As an immediate consequence we obtain that $M_{0} \rightarrow+\infty, r_{1} \rightarrow 0, s_{1} \rightarrow 0$, since $M_{0}>\tilde{M}_{0}=$ $r_{k}^{\frac{2}{p_{\varepsilon}-1}} M_{0}$ and $0<r_{1}<s_{1}<\tilde{s}_{1}=\frac{s_{1}}{r_{k}}$. We divide the remaining part of the proof in three steps:

Step 1: $\tilde{M}_{k}$ is bounded.

Let us consider the energy-like functionals

$$
\begin{aligned}
& \tilde{H}_{\lambda}(r):=\frac{\left(\tilde{u}_{\varepsilon, k}^{\prime}(r)\right)^{2}}{2}+\frac{\left|\tilde{u}_{\varepsilon, k}(r)\right|^{p_{\varepsilon}+1}}{\lambda\left(p_{\varepsilon}+1\right)}, \\
& \tilde{H}_{\Lambda}(r):=\frac{\left(\tilde{u}_{\varepsilon, k}^{\prime}(r)\right)^{2}}{2}+\frac{\left|\tilde{u}_{\varepsilon, k}(r)\right|^{p_{\varepsilon}+1}}{\Lambda\left(p_{\varepsilon}+1\right)} .
\end{aligned}
$$

If $k+1$ is even, then $\tilde{H}_{\lambda}(r)$ is monotone decreasing in $\left[\tilde{s}_{k-1}, \tilde{s}_{k}\right]$. Then $\tilde{H}_{\lambda}\left(\tilde{s}_{k-1}\right) \geq \tilde{H}_{\lambda}\left(\tilde{s}_{k}\right)$ from which we deduce that

$$
\tilde{M}_{k-1} \geq \tilde{M}_{k}
$$

If $k+1$ is odd then $\tilde{H}_{\lambda}(r)$ is monotone decreasing in $\left[\tilde{s}_{k-1}, \tilde{t}_{k-1}\right]$, while $\tilde{H}_{\Lambda}(r)$ is monotone decreasing in $\left[\tilde{t}_{k-1}, \tilde{s}_{k}\right]$. Hence, using that $\lambda \leq \Lambda$, we have

$$
\tilde{H}_{\lambda}\left(\tilde{s}_{k-1}\right) \geq \tilde{H}_{\lambda}\left(\tilde{t}_{k-1}\right) \geq \tilde{H}_{\Lambda}\left(\tilde{t}_{k-1}\right) \geq \tilde{H}_{\Lambda}\left(\tilde{s}_{k}\right) .
$$

From this we easily deduce that

$$
\tilde{M}_{k-1} \geq\left(\frac{\lambda}{\Lambda}\right)^{\frac{1}{p_{\varepsilon}+1}} \tilde{M}_{k}
$$


Exploiting the inductive hypothesis we deduce from (6.2)-(6.3) that $\tilde{M}_{k}$ is bounded.

Step 2: $\liminf _{\varepsilon \rightarrow 0} r_{k}>0$.

Assume by contradiction that $r_{k} \rightarrow 0$, for some subsequence $\varepsilon \rightarrow 0$. Then, the limit domain of $A_{\tilde{r}_{1}, \frac{1}{r_{k}}}$ is $\mathbb{R}^{N} \backslash\{0\}$ and, in view of Step 1 and the inductive hypothesis, we have that the restriction $\left.\tilde{u}_{\varepsilon, k}\right|_{\tilde{\tilde{r}}_{1}, \frac{1}{r_{k}}}$ is uniformly bounded. Hence $\tilde{u}_{\varepsilon, k} \rightarrow \tilde{u}_{k}$ in $C_{l o c}^{2}\left(\mathbb{R}^{N} \backslash\{0\}\right)$, where $\tilde{u}_{k}$ is a radial solution of

$$
-\mathcal{M}_{\lambda, \Lambda}^{-}\left(D^{2} u\right)=|u|^{p_{-}^{*}-1} u \quad \text { in } \mathbb{R}^{N} \backslash\{0\} .
$$

Moreover, $\tilde{u}_{k}$ is non-trivial and sign-changing because by construction we have $\tilde{u}_{k} \equiv \overline{\tilde{u}}_{k}$ in $\bar{B} \backslash\{0\}$, where $\overline{\tilde{u}}_{k}$ is the limit of $\left.\tilde{u}_{\varepsilon, k}\right|_{B}$. In particular, $\tilde{u}_{k}^{\prime}(1) \neq 0, \tilde{u}_{k}(1)=0$ and thus it cannot happen that $\tilde{u}_{k} \equiv 0$ in $\mathbb{R}^{N} \backslash \bar{B}$. Therefore, the restriction of $\tilde{u}_{k}$ to $\mathbb{R}^{N} \backslash \bar{B}$ is a constant-sign radial solution of

$$
\left\{\begin{array}{cl}
-\mathcal{M}_{\lambda, \Lambda}^{-}\left(D^{2} u\right)=|u|^{p_{-}^{*}-1} u & \text { in } \mathbb{R}^{N} \backslash \bar{B}, \\
u=0 & \text { on } \partial B .
\end{array}\right.
$$

This contradicts [11, Theorem 1.1].

Step 3: conclusion.

In view of Step 1 and Step 2 , since $\tilde{M}_{k}=r_{k}^{\frac{2}{p_{\varepsilon}-1}} M_{k}$, we infer that $M_{k}$ is bounded. Moreover it is bounded away from zero. Indeed, arguing as in Remark 4.2 we get that $M_{k}^{p_{\varepsilon}-1} \geq \lambda_{1}^{+}\left(-\mathcal{M}_{\lambda, \Lambda}^{+} ; B\right)$ if $k+1$ is odd, or $M_{k}^{p_{\varepsilon}-1} \geq \lambda_{1}^{+}\left(-\mathcal{M}_{\lambda, \Lambda}^{-} ; B\right)$ if $k+1$ is even.

Up to a subsequence we then have $M_{k} \rightarrow \bar{M}_{k}, r_{k} \rightarrow \bar{r}_{k}$, for some $\bar{M}_{k} \in(0,+\infty), \bar{r}_{k} \in(0,1]$. Arguing as in the proof of Theorem 5.5, taking the restriction of $u_{\varepsilon}$ to $A_{r_{k}, 1}$, exploiting that $M_{k}$ is bounded and that $\lambda_{1}^{+}\left(-\mathcal{M}_{\lambda, \Lambda}^{ \pm} ; A_{r_{k}, 1}\right) \rightarrow+\infty$ if $r_{k} \rightarrow 1$, we infer that $\bar{r}_{k} \neq 1$.

Now, using the definitions of $\tilde{M}_{i}, \tilde{r}_{i}, \tilde{s}_{i}$ for $i=2, \ldots, k-1$ and the results proved in the first part of the proof, we conclude that $M_{i} \rightarrow \bar{M}_{i}=\bar{r}_{k}^{-\frac{2}{p_{-}^{*-1}}} \bar{M}_{i} \in(0,+\infty), r_{i} \rightarrow \bar{r}_{i}=\bar{r}_{i} \bar{r}_{k}$, $s_{i} \rightarrow \bar{s}_{i}=\overline{\tilde{s}}_{i} \bar{r}_{k}$ for $i=2, \ldots, k-1$, and it holds

$$
0<\bar{r}_{2}<\bar{s}_{2}<\ldots<\bar{r}_{k-1}<\bar{s}_{k-1} .
$$

Summing up, since the restriction of $u_{\varepsilon}$ to $A_{r_{1}, 1}$ is uniformly bounded and $\bar{r}_{k} \neq 1$ we deduce that, up to a subsequence, $u_{\varepsilon} \rightarrow \bar{u}$ in $C_{l o c}^{2}(\bar{B} \backslash\{0\})$, where $\bar{u}$ is a non-trivial radial sign-changing solution of

$$
\left\{\begin{array}{cl}
-\mathcal{M}_{\lambda, \Lambda}^{-}\left(D^{2} u\right)=|u|^{p_{-}^{*}-1} u & \text { in } B \backslash\{0\} \\
u=0 & \text { on } \partial B
\end{array}\right.
$$

with $k$ nodal regions. Since $\bar{r}_{k} \in(0,1), \overline{\widetilde{s}_{k-1}} \in(0,1)$ we infer that $\bar{s}_{k-1}<\bar{r}_{k}$. Moreover, denoting by $\bar{s}_{k}$ the limit point of $s_{k}$, from the regularity of $\bar{u}$ in compact subsets of $\bar{B} \backslash\{0\}$ and since $\bar{M}_{k} \neq 0$, we deduce that it cannot happen that $\bar{s}_{k}=\bar{r}_{k}$. Moreover, since $u_{\varepsilon}^{\prime}\left(s_{k}\right)=0$ and $\left|\bar{u}^{\prime}(1)\right|>0$ in view of the Hopf's Lemma, we infer that $s_{k} \rightarrow 1$ cannot happen. Therefore the nodes and the extrema of $\bar{u}$ are ordered in the following way

$$
0<\bar{r}_{2}<\bar{s}_{2}<\ldots<\bar{r}_{k-1}<\bar{s}_{k-1}<\bar{r}_{k}<\bar{s}_{k}<1,
$$

as expected. 
At the end, arguing as in the proof of Theorem [5.5 we see that $\bar{u}(x)=\bar{r}_{k}^{-\frac{2}{p_{-}^{*}-1}} \bar{u}_{k}\left(\bar{r}_{k} x\right)$, for $x \in B_{\bar{r}_{k}} \backslash\{0\}$. Hence $\bar{u}$ extends to a $C^{2}$ radial function near the origin and it is a sign-changing solution of (1.6) having $k$ nodal regions. This completes the proof of the inductive step.

\section{ASYMPtotic ANALYSis of RADIAL Sign-CHANGING SOLUTIONS TO (1.10) With TWO NODAL REGIONS}

Let $u_{n}$ be a sequence of radial sign-changing solutions to (1.10) with two nodal domains, where $p_{n}=p_{+}^{* *}-\varepsilon_{n}, \varepsilon_{n} \searrow 0$ as $n \rightarrow+\infty$. The first result is about the behavior of $M_{n}:=\left\|u_{n}\right\|_{\infty}$ as $n \rightarrow+\infty$. We set $M_{0}=M_{0}(n):=\left\|u_{n}^{+}\right\|_{\infty}=u_{n}(0)$ and $M_{1}=M_{1}(n):=\left\|u_{n}^{-}\right\|_{\infty}$, and we denote by $r_{1}=r_{1}(n)$ the nodal radius and by $s_{1}=s_{1}(n)$ the minimum point.

Proposition 7.1. We have $M_{n} \rightarrow+\infty$, as $n \rightarrow+\infty$.

Proof. Arguing as in Remark 4.2 we infer that

$$
\begin{aligned}
& M_{0}^{p_{n}-1} \geq \lambda_{1}^{+}\left(-\mathcal{M}_{\lambda, \Lambda}^{+} ; B_{r_{1}}\right) \geq \lambda_{1}^{+}\left(-\mathcal{M}_{\lambda, \Lambda}^{+} ; B\right), \\
& M_{1}^{p_{n}-1} \geq \lambda_{1}^{+}\left(-\mathcal{M}_{\lambda, \Lambda}^{-} ; A_{r_{1}, 1}\right) \geq \lambda_{1}^{+}\left(-\mathcal{M}_{\lambda, \Lambda}^{-} ; B\right),
\end{aligned}
$$

which readily implies that $M_{0}$ and $M_{1}$ are uniformly bounded from below away from zero. Since $M_{n}=\max \left\{M_{0}, M_{1}\right\}$ the same holds for $M_{n}$.

Now, assume that $M_{n} \rightarrow \bar{M} \in(0,+\infty)$ for some subsequence. Then, from elliptic regularity estimates we deduce that $u_{n}$ is uniformly bounded in $C^{2}(\bar{B})$. In addition, in view of (7.1) it cannot happen that $r_{1} \rightarrow 0$ or $r_{1} \rightarrow 1$, otherwise $\lambda_{1}^{+}\left(-\mathcal{M}_{\lambda, \Lambda}^{+}, B_{r_{1}}\right) \rightarrow+\infty$ or $\lambda_{1}^{+}\left(-\mathcal{M}_{\lambda, \Lambda}^{-} ; A_{r_{1}, 1}\right) \rightarrow+\infty$ and by (7.1) this would imply that one between $M_{0}$ and $M_{1}$ blows-up, contradicting the uniform boundedness of $u_{n}$.

Therefore $r_{1} \nrightarrow \rightarrow 0, r_{1} \nrightarrow \nrightarrow 1$ and by regularity estimates, up to a further subsequence, as $n \rightarrow+\infty$, we have $u_{n} \rightarrow \bar{u}$ in $C^{2}(\bar{B})$, where $\bar{u}$ is a radial sign-changing solution of (1.11) and $\bar{u}(0)>0$ (because of (7.1) $)$, but this contradicts Proposition 3.4

Proposition 7.2. We have:

i) $0<\liminf _{n \rightarrow+\infty} r_{1} M_{0}^{\frac{p_{n}-1}{2}} \leq \limsup _{n \rightarrow+\infty} r_{1} M_{0}^{\frac{p_{n}-1}{2}}<+\infty$;

ii) $0<\liminf _{n \rightarrow+\infty} \frac{M_{0}}{M_{1}} \leq \limsup _{n \rightarrow+\infty} \frac{M_{0}}{M_{1}}<+\infty$;

iii) $M_{0} \rightarrow+\infty, M_{1} \rightarrow+\infty$;

iv) $r_{1} \rightarrow 0$;

v) the rescaled function

$$
\tilde{u}_{n}(x):=r_{1}^{\frac{2}{p_{n}-1}} u_{n}\left(r_{1} x\right), x \in B_{1 / r_{1}},
$$

converges, up to a subsequence, in $C_{\text {loc }}^{2}\left(\mathbb{R}^{N}\right)$ to a non-trivial radial sign-changing solution of

$$
-\mathcal{M}_{\lambda, \Lambda}^{+}\left(D^{2} u\right)=|u|^{p_{+}^{* *}-1} u \text { in } \mathbb{R}^{N}
$$

vi) $s_{1} \rightarrow 0$

Proof. If along a subsequence it holds that $r_{1} M_{0}^{\frac{p_{n}-1}{2}} \rightarrow+\infty$, then, defining the rescaled function

$$
\hat{u}_{n}(x):=\frac{1}{M_{0}} u_{n}\left(\frac{x}{M_{0}^{\frac{p_{n}-1}{2}}}\right), x \in B_{M_{0}^{\frac{p_{n}-1}{2}}},
$$


we have $\hat{u}_{n}(0)=1$ and by the usual argument we infer that $\hat{u}_{n}^{+} \rightarrow \hat{u}$ in $C_{l o c}^{2}\left(\mathbb{R}^{N}\right)$, where $\hat{u}$ is a positive radial solution of

$$
-\mathcal{M}_{\lambda, \Lambda}^{+}\left(D^{2} u\right)=u^{p_{+}^{* *}} \text { in } \mathbb{R}^{N} .
$$

On the other hand, since $p_{+}^{* *}<p_{+}^{*}$ then by [9, Theorem 1.1] we get that (7.4) does not have positive radial solutions, and this gives a contradiction.

If along a subsequence it holds that $r_{1} M_{0}^{\frac{p_{n}-1}{2}} \rightarrow 0$, arguing as in Remark 4.2 for $\hat{u}_{n}$, we would have

$$
1 \geq \lambda_{1}^{+}\left(-\mathcal{M}_{\lambda, \Lambda}^{+}, B_{r_{1} M_{0}^{\frac{p_{n}-1}{2}}}\right) \rightarrow+\infty
$$

which is a contradiction. This completes the proof of i).

To prove ii) we show that $\frac{M_{0}}{M_{1}} \rightarrow 0$ and $\frac{M_{0}}{M_{1}} \rightarrow+\infty$ cannot occur along any subsequence.

Let us consider the functionals

$$
H_{\lambda}(r):=\frac{\left(u_{n}^{\prime}\right)^{2}(r)}{2}+\frac{u_{n}^{p_{n}+1}}{\lambda\left(p_{n}+1\right)}, \quad r \in\left[0, t_{0}\right], \quad H_{\Lambda}(r):=\frac{\left(u_{n}^{\prime}\right)^{2}(r)}{2}+\frac{\left|u_{n}\right|^{p_{n}+1}}{\Lambda\left(p_{n}+1\right)}, \quad r \in\left[t_{0}, s_{1}\right],
$$

where $t_{0} \in\left(0, r_{1}\right)$ is the only radius such that $u_{n}^{\prime \prime}<0$ in $\left(0, t_{0}\right)$ and $u_{n}^{\prime \prime}>0$ in $\left(t_{0}, r_{1}\right)$. Exploiting the ODE satisfied by $u_{n}$ in $\left[0, s_{1}\right]$, we check that $H_{\lambda}$ is decreasing in $\left[0, t_{0}\right]$ and $H_{\Lambda}$ is decreasing in $\left[t_{0}, s_{1}\right]$. Moreover, since $\lambda \leq \Lambda$ and $u_{n}\left(t_{0}\right)>0$ we infer that $H_{\lambda}\left(t_{0}\right) \geq H_{\Lambda}\left(t_{0}\right)$. Summing up we get that

$$
H_{\lambda}(0) \geq H_{\lambda}\left(t_{0}\right) \geq H_{\Lambda}\left(t_{0}\right) \geq H_{\Lambda}\left(s_{1}\right),
$$

and since $H_{\lambda}(0)=\frac{M_{0}^{p_{n}+1}}{\lambda\left(p_{n}+1\right)}, H_{\Lambda}\left(s_{1}\right)=\frac{M_{1}^{p_{n}+1}}{\Lambda\left(p_{n}+1\right)}$ we deduce that

$$
\frac{M_{0}}{M_{1}} \geq\left(\frac{\lambda}{\Lambda}\right)^{\frac{1}{p_{n}+1}} \text {. }
$$

From (7.5) it follows that

and this implies

$$
M_{n} \leq\left(\frac{\Lambda}{\lambda}\right)^{\frac{1}{p_{n}+1}} M_{0}
$$

$$
\lim _{n \rightarrow+\infty} M_{0}=+\infty \text {. }
$$

Assume now that, for some subsequence, $\frac{M_{0}}{M_{1}} \rightarrow+\infty$ and consider again the rescaled function $\hat{u}_{n}$. By construction $\hat{u}_{n}(0)=1,\left\|\hat{u}_{n}\right\|_{\infty} \leq 1$ and as before, up to a subsequence as $n \rightarrow+\infty$, we have $\hat{u}_{n} \rightarrow \hat{u}$ in $C_{l o c}^{2}\left(\mathbb{R}^{N}\right)$, where $\hat{u}$ is a non-trivial radial solution to (7.3) satisfying $\hat{u}(0)=1$.

From i) there exists $c_{1}>0$ such that $\hat{u}>0$ in $B_{c_{1}}$ and $\hat{u}=0$ on $\partial B_{c_{1}}$. Moreover $\hat{u}^{\prime}\left(c_{1}\right)<0$. On the other hand, taking into account that $\frac{M_{0}}{M_{1}} \rightarrow+\infty$, we deduce that $\hat{u} \equiv 0$ in $\mathbb{R}^{N} \backslash \bar{B}_{c_{1}}$, since for any fixed $x$ such that $|x|>c_{1}$ and for all sufficiently large $n$ we have

$$
\left|\hat{u}_{n}(x)\right|=\left|\frac{1}{M_{0}} u_{n}\left(\frac{x}{M_{0}^{\frac{p_{n}-1}{2}}}\right)\right| \leq \frac{M_{1}}{M_{0}} .
$$

Passing to the limit as $n \rightarrow+\infty$ we obtain $\hat{u}(x)=0$, which contradicts the $C^{1}$ regularity of $\hat{u}$.

Statement iii) is an immediate consequence of (7.6) and ii), while iv) directly follows from i) and iii).

Let us prove v) and vi). From i) and ii) the rescaled function $\tilde{u}_{n}$ in (7.2) is uniformly bounded, and by iii) the limit of $B_{1 / r_{1}}$ is $\mathbb{R}^{N}$. Moreover, by construction $\left.\tilde{u}_{n}\right|_{B}=v_{p_{n},+}$ is the only radial positive solution of (1.10). Hence, up to a subsequence as $n \rightarrow+\infty$, we have $\tilde{u}_{n} \rightarrow \tilde{u}$ in $C_{\text {loc }}^{2}\left(\mathbb{R}^{N}\right)$, where $\tilde{u}$ is a non-trivial radial solution of (7.3), and this proves v). In particular $\tilde{u}>0$ in $B$, 
$\tilde{u}=0$ on $\partial B$ and the function $\tilde{u}^{-}=\tilde{u}^{-}(r)$ coincides, for $r>1$, with the unique maximal solution of (2.7) with $p=p_{+}^{* *}, \alpha=-\left(\tilde{u}^{+}\right)^{\prime}(1)$. Now arguing as in the proof of the first part of Proposition 4.4. we infer that $\frac{s_{1}}{r_{1}}$ is bounded. Since $r_{1} \rightarrow 0$ by iv), we conclude that $s_{1} \rightarrow 0$ as well.

In the next result we state and prove a uniform upper bound that will be crucial in the sequel (see Sect. 10).

Proposition 7.3. Let $\tilde{u}_{n}^{-}$be the negative part of the rescaled function $\tilde{u}_{n}$ defined in (7.2). Then, up to a subsequence as $n \rightarrow+\infty, \tilde{u}_{n}^{-} \rightarrow W_{-}$in $C_{l o c}^{2}\left(\mathbb{R}^{N} \backslash B\right)$, where $W_{-}$is the only positive radial fast decaying solution of (1.12). Moreover, there exist two positive constants $C, K$ (independent on $n$ ) such that for all sufficiently large $n$ it holds

$$
\tilde{u}_{n}^{-}(r) \leq \frac{C}{\left(r^{2}-\left(\tilde{t}_{1}\right)^{2}+K\right)^{\frac{\tilde{N}_{-}-2}{2}}} \quad \text { for } r \in\left[\tilde{t}_{1}, \frac{1}{r_{1}}\right],
$$

where $\tilde{t}_{1} \rightarrow \bar{t}_{1} \in(1,+\infty)$ and $\bar{t}_{1}$ is the only radius where $W_{-}=W_{-}(r)$ changes concavity.

Proof. In view of Proposition $7.2 \mathrm{v}$ ), up to a subsequence as $n \rightarrow+\infty$, we have in particular that $\tilde{u}_{n}^{-} \rightarrow \tilde{u}^{-}$in $C_{l o c}^{2}\left(\mathbb{R}^{N} \backslash B\right)$, where $\tilde{u}^{-}$is a positive radial solution of (1.12). We claim that $\tilde{u}^{-}$ is fast decaying. By (ii) of Theorem 2.2 this is equivalent to show that $\alpha_{-}^{*}\left(p_{+}^{* *}\right)=\left(\tilde{u}^{-}\right)^{\prime}(1)$. To prove this, we observe that $\tilde{u}_{n}^{-}=\tilde{u}_{n}^{-}(r)$ is a solution of (2.7) with $p=p_{n}, \alpha=\alpha(n)=\left(\tilde{u}_{n}^{-}\right)^{\prime}(1)=$ $-v_{p_{n},+}^{\prime}(1)$ and such that $\tilde{u}_{n}^{-}\left(1 / r_{1}\right)=0$, and thus, by definition of $\alpha_{-}^{*}$, we have

$$
\alpha_{-}^{*}\left(p_{n}\right) \leq\left(\tilde{u}_{n}^{-}\right)^{\prime}(1)
$$

Then, passing to the limit as $n \rightarrow+\infty$, exploiting Proposition 2.3 and taking into account that $\tilde{u}_{n} \rightarrow \tilde{u}$ in $C_{l o c}^{2}\left(\mathbb{R}^{N}\right)$, we deduce that

$$
\alpha_{-}^{*}\left(p_{+}^{* *}\right) \leq\left(\tilde{u}^{-}\right)^{\prime}(1)
$$

On the other hand, since $\tilde{u}^{-}=\tilde{u}^{-}(r)$ is defined and positive in the whole $(1,+\infty)$, then, by (i) of Theorem 2.2 we infer that $\alpha_{-}^{*}\left(p_{+}^{* *}\right) \geq\left(\tilde{u}^{-}\right)^{\prime}(1)$. Hence, $\tilde{u}^{-}=W_{-}$is the only positive radial fast decaying solution of (1.12).

For (7.7), we notice that it is exactly inequality (2.13) obtained in the proof of Proposition 2.3. Indeed, denoting by $\tilde{t}_{1}$ the only radius where $\tilde{u}_{n}^{-}=\tilde{u}_{n}^{-}(r)$ changes concavity and setting $v_{n}(r):=\tilde{u}_{n}^{-}(r)$, we have that $v_{n}$ satisfies (2.14) in $\left[\tilde{t}_{1}, \frac{1}{r_{1}}\right]$, with $p_{n}=p_{+}^{* *}-\varepsilon_{n}$. In the present case, $p_{n} \rightarrow p_{+}^{* *}$, which still satisfies $p_{+}^{* *}>p_{-}^{*}>\frac{\tilde{N}_{-}+2}{\tilde{N}_{-}-2}$. Then the proof follows verbatim, taking into account that $v_{n} \rightarrow W_{-}$in $C_{l o c}^{2}\left([1,+\infty)\right.$, and that $\tilde{t}_{1} \rightarrow \bar{t}_{1}, v_{n}\left(\tilde{t}_{1}\right) \rightarrow W_{-}\left(\bar{t}_{1}\right)$, as $n \rightarrow+\infty$, where $\bar{t}_{1}$ is the only radius where $W_{-}=W_{-}(r)$ changes concavity.

We conclude this section by studying the limiting behavior of $u_{n}$.

Proposition 7.4. Up to a subsequence, as $n \rightarrow+\infty$, it holds that $u_{n} \rightarrow 0$ in $C_{l o c}^{2}(\bar{B} \backslash\{0\})$.

Proof. The proof is carried out along the same line of [4, Theorem 1.1, ii)]. By elliptic estimates, it is sufficient to show that for any fixed $\rho \in(0,1)$

$$
\left\|u_{n}\right\|_{\infty, A_{\rho}} \rightarrow 0 \quad \text { as } n \rightarrow+\infty
$$

where $A_{\rho}=\{x \in \bar{B}:|x| \geq \rho\}$ and $\|\cdot\|_{\infty, A_{\rho}}$ denotes the $L^{\infty}$-norm in $A_{\rho}$. Using (7.7) and the definition (7.2) of $\tilde{u}_{n}$ we have

$$
r_{1}^{\frac{2}{p_{n}-1}} u_{n}^{-}\left(r_{1} r\right) \leq C \frac{1}{\left(r^{2}-\frac{t_{1}^{2}}{r_{1}^{2}}+K\right)^{\frac{\tilde{N}_{-}-2}{2}}} \quad \text { for } r \in\left[\frac{t_{1}}{r_{1}}, \frac{1}{r_{1}}\right),
$$


or equivalently

$$
r_{1}^{\frac{2}{p_{n}-1}} u_{n}^{-}(r) \leq C \frac{1}{\left(\frac{r^{2}}{r_{1}^{2}}-\frac{t_{1}^{2}}{r_{1}^{2}}+K\right)^{\frac{\tilde{N}_{-}-2}{2}}}, \quad \text { for } r \in\left[t_{1}, 1\right)
$$

for some positive constants $C$ and $K$ independent on $n$.

Now, since $\frac{t_{1}}{r_{1}} \rightarrow \bar{t}_{1} \in(1,+\infty)$ by Proposition 7.3 and $r_{1} \rightarrow 0$ in view of Proposition 7.2 iv), we infer that $t_{1} \rightarrow 0$. Hence for sufficiently large $n$ we have $t_{1}^{2} \leq \frac{\rho^{2}}{2}$ and

$$
\left\|u_{n}\right\|_{\infty, A_{\rho}}=\left\|u_{n}^{-}\right\|_{\infty, A_{\rho}} \leq \frac{C}{\left(\frac{\rho^{2}}{2}\right)^{\frac{\tilde{N}_{-}-2}{2}}} r_{1}^{\tilde{N}_{-}-2-\frac{2}{p_{n}-1}} .
$$

Then the conclusion follows since $r_{1} \rightarrow 0$ and $\tilde{N}_{-}-2-\frac{2}{p_{n}-1} \rightarrow \tilde{N}_{-}-2-\frac{2}{p_{+}^{* *}-1}>0$.

Remark 7.5. By Proposition 7.2 and Proposition 7.4 we have that the maximum and the minimum of $u_{n}$, namely $M_{0}$ and $-M_{1}$, blow up at the same rate and the minimum point $s_{1}$ converges to the maximum point which is zero. Thus we have concentration of the positive and negative part at the same point.

This is a new phenomenon, as compared with the classical Lane-Emden problem in which case, whenever the rate of blow-up of the positive and negative part is the same, the two nodal regions separate and the concentration points of the negative and positive part are different (see [3]).

\section{Asymptotic analysis of Radial Sign-Changing Solutions to (1.10) With three NODAL REGIONS}

Having proved in the previous section Theorem 1.4 when $k=2$ we could argue by induction to get the general result. However, since passing from even to odd the statement changes, we prefer to detail the proof for $k=3$, for the reader convenience.

Let $u_{n}$ be a sign-changing solution of (1.10) with three nodal regions, let $r_{i}=r_{i}(n), i=1,2$, be the nodal radii, let $s_{i}=s_{i}(n), i=1,2$, be the maximum points of $\left|u_{n}\right|$ in the second and third nodal region, and denote by $M_{i}=M_{i}(n), i=0,1,2$ the maximum values of $\left|u_{n}\right|$ in each nodal region.

Proposition 8.1. Up to a subsequence, as $n \rightarrow+\infty$, we have: $M_{0} \rightarrow+\infty, M_{1} \rightarrow+\infty, r_{i} \rightarrow 0$, $s_{i} \rightarrow 0$ for $i=1,2, M_{2} \rightarrow \bar{M}$, for some $\bar{M}>0$, and $u_{n} \rightarrow \bar{v}$ in $C_{l o c}^{2}(\bar{B} \backslash\{0\})$, where $\bar{v}$ is the unique positive radial solution of (1.11).

Proof. Let us consider the rescaled function

$$
\tilde{u}_{n}(x):=r_{2}^{\frac{2}{p_{n}-1}} u_{n}\left(r_{2} x\right), \quad x \in B_{1 / r_{2}} .
$$

Then the restriction of $\tilde{u}_{n}$ to the unit ball $B$ is the radial sign-changing solution of (1.10) with two nodal regions so that the results of Sect. 7 apply. In particular, up to a subsequence, as $n \rightarrow+\infty$, we have:

$$
\frac{r_{1}}{r_{2}} \rightarrow 0, \quad r_{2}^{\frac{2}{p_{n}-1}} M_{i} \rightarrow+\infty \quad \text { for } i=0,1
$$

and

$$
\frac{M_{0}}{M_{1}}=\frac{r_{2}^{\frac{2}{p_{n}-1}} M_{0}}{r_{2}^{\frac{2}{p_{n}-1}} M_{1}} \rightarrow c_{0}
$$


for some positive constant $c_{0}$. From 8.2 we deduce that

$$
\begin{aligned}
M_{i} & \rightarrow+\infty \quad \text { for } i=0,1 \\
r_{1} & \rightarrow 0 .
\end{aligned}
$$

Moreover

$$
\tilde{H}(r)=\frac{\left(\tilde{u}_{n}^{\prime}(r)\right)^{2}}{2}+\frac{\left|\tilde{u}_{n}(r)\right|^{p_{n}+1}}{\lambda\left(p_{n}+1\right)}
$$

is monotone decreasing in $\left[1, \frac{s_{2}}{r_{2}}\right]$, hence

$$
\frac{\left(r_{2}^{\frac{2}{p_{n}-1}} M_{2}\right)^{p_{n}+1}}{\lambda\left(p_{n}+1\right)} \leq \frac{\left(\tilde{u}_{n}^{\prime}(1)\right)^{2}}{2} .
$$

Since $\tilde{u}_{n}^{\prime}(1) \rightarrow 0$, then

$$
r_{2}^{\frac{2}{p_{n}-1}} M_{2} \rightarrow 0
$$

and, using the lower bound $M_{2}^{p_{n}-1} \geq \lambda_{1}^{+}\left(-\mathcal{M}_{\lambda, \Lambda}^{+} ; B\right.$ ) (see (17.1)), we also deduce that $r_{2} \rightarrow 0$ and, as a consequence, $s_{1} \rightarrow 0$. Putting together (8.2) for $i=1$ and (8.5) we have

$$
\frac{M_{1}}{M_{2}}=\frac{r_{2}^{\frac{2}{p_{n}-1}} M_{1}}{r_{2}^{\frac{2}{p_{n}-1}} M_{2}} \rightarrow+\infty
$$

Arguing as in Proposition 4.4 and Corollary 4.5 we also deduce that $s_{2} \rightarrow 0$. If $M_{2}$ is bounded from above, then $\left.u_{n}\right|_{A_{r_{2}, 1}} \rightarrow \bar{u}$ in $C_{\text {loc }}^{2}(\bar{B} \backslash\{0\})$ for some radial positive function $\bar{u}$. Since $s_{2} \rightarrow 0$, then as in the proof of Theorem 4.9 we obtain that $\bar{u}$ extends to the unique positive radial solution of (1.11), as we aim to prove. Hence to complete the proof it remains to show that $\limsup _{n \rightarrow+\infty} M_{2}<+\infty$. On the contrary, let us assume that, along some sequence $n \rightarrow+\infty$, $M_{2} \rightarrow+\infty$ and consider the rescaled function

$$
\hat{u}_{n}(x):=\frac{1}{M_{2}} u_{n}\left(\frac{x}{M_{2}^{\frac{p_{n}-1}{2}}}\right), x \in A_{r_{2} M_{2}^{\frac{p_{n}-1}{2}}, M_{2}^{\frac{p_{n}-1}{2}}} .
$$

Since the limit domain of $\hat{u}_{n}$ is $\mathbb{R}^{N} \backslash\{0\}$ by (8.5), we can argue exactly as in Proposition 4.8 to deduce that $\hat{u}_{n} \rightarrow \hat{u}$ in $C_{l o c}^{2}\left(\mathbb{R}^{N} \backslash\{0\}\right)$, where $\hat{u}$ can be extended to a non-trivial radial positive solution to (7.4). This is clearly a contradiction since $p_{+}^{* *}<p_{+}^{*}$. Hence the only possibility is that $M_{2} \rightarrow \bar{M}$ for some positive constant $\bar{M}$ as we wanted to show.

\section{Asymptotic AnAlysis of RAdiAl Sign-CHANGing SOLUTions to (1.10) With $k$ NODAL REGIONS}

In this section we prove Theorem 1.4

Proof of Theorem 1.4. We argue by induction on $k$. The steps $k=2,3$ have been proved respectively in Sect. 7 and Sect. 8. So let us assume that the assertion holds true for solutions with $k$ nodal regions and let $u_{n}$ be a radial sign-changing solution to (1.10) with $k+1$ nodal regions.

Case 1: if $k+1$ is even, then the restriction to $B$ of the rescaled function

$$
\tilde{u}_{n, k}(x):=r_{k}^{\frac{2}{p_{n}-1}} u_{n}\left(r_{k} x\right), \quad x \in B_{1 / r_{k}}
$$

is a solution to (1.10) having $k$ nodal regions, with $k$ odd. Hence, exploiting the inductive hypothesis and the definition of $\tilde{u}_{n, k}$, we infer that, up to a subsequence as $n \rightarrow+\infty, M_{0} r_{k}^{\frac{2}{p_{n}-1}} \rightarrow$ $+\infty, \ldots, M_{k-2} r_{k}^{\frac{2}{p_{n}-1}} \rightarrow+\infty$, and $M_{k-1} r_{k}^{\frac{2}{p_{n}-1}} \rightarrow \bar{M}$, for some $\bar{M}>0$. This readily implies that 
$M_{0} \rightarrow+\infty, \ldots, M_{k-2} \rightarrow+\infty$. Moreover, by inductive hypothesis, we also have $\frac{r_{i}}{r_{k}} \rightarrow 0, \frac{s_{i}}{r_{k}} \rightarrow 0$ for $i=1, \ldots, k-1$, which easily implies that $r_{i} \rightarrow 0, s_{i} \rightarrow 0$, for $i=1, \ldots, k-1$.

Finally, exploiting again the inductive hypothesis we deduce that $\frac{M_{2 j}}{M_{2 j+1}}=\frac{M_{2 j} r_{k}^{\frac{p_{n}-1}{2}}}{M_{2 j} r_{k}^{\frac{p n}{2}}} \rightarrow c_{j}$ for $j=0, \ldots, \frac{k-3}{2}$, with $c_{j}$ positive constants, and that $\frac{M_{2 j+1}}{M_{2 j+2}}=\frac{M_{2 j+1} r_{k} \frac{p_{n-1}-1}{p_{p-1}}}{M_{2 j+2} r_{k}^{2}} \rightarrow+\infty$ for $j=$ $0, \ldots, \frac{k-3}{2}$.

Now, arguing as in the proof of (7.5) we have that

$$
\frac{M_{k-1}}{M_{k}} \geq\left(\frac{\lambda}{\Lambda}\right)^{\frac{1}{p_{n}+1}}
$$

Let us show that

$$
\limsup _{n \rightarrow+\infty} \frac{M_{k-1}}{M_{k}}<+\infty \text {. }
$$

Assume by contradiction that, for a subsequence, $\frac{M_{k-1}}{M_{k}} \rightarrow+\infty$ and consider the restriction of $\tilde{u}_{n, k}$ to the annulus $A_{r_{k-1} / r_{k}, 1 / r_{k}}$. Since $M_{k-1} r_{k}^{\frac{2}{p_{n}-1}} \rightarrow \bar{M}$, for some $\bar{M}>0$ then from (9.1) we deduce that $\left.\tilde{u}_{n, k}\right|_{A_{r_{k-1} / r_{k}, 1 / r_{k}}}$ is uniformly bounded. We claim that $r_{k} \not \rightarrow 1$. Otherwise, since $\left.\tilde{u}_{\varepsilon, k}\right|_{A_{1,1 / r_{k}}}$ is uniformly bounded and $r_{k} \rightarrow 1$ we would have $\lambda_{1}\left(-\mathcal{M}_{\lambda, \Lambda}^{-} ; A_{1,1 / r_{k}}\right) \rightarrow+\infty$, and from (4.2) we would have a contradiction. Hence $r_{k} \rightarrow \bar{r} \in(0,1)$ or $r_{k} \rightarrow 0$, and recalling that by inductive hypothesis $\frac{r_{k-1}}{r_{k}} \rightarrow 0$, we infer that $\left.\tilde{u}_{n, k}\right|_{A_{r_{k-1} / r_{k}, 1 / r_{k}}} \rightarrow \tilde{u}$ in $C_{l o c}^{2}(\Pi)$, where $\tilde{u}$ is non-trivial (because by inductive hypothesis it coincides with $\bar{u}$ in $B$ ) and the limit domain $\Pi$ is either $\mathbb{R}^{N} \backslash\{0\}$ or $\bar{B}_{\frac{1}{\tilde{r}}} \backslash\{0\}$. Since we are assuming that $\frac{M_{k-1}}{M_{k}} \rightarrow+\infty$, arguing as in the proof of Proposition 7.2, ii), we deduce that $\tilde{u} \equiv 0$ in $\Pi \cap\left\{x \in \mathbb{R}^{N} ;|x|>1\right\}$ contradicting the regularity of $\tilde{u}$. Hence the only possibility is

$$
\frac{M_{k-1}}{M_{k}} \rightarrow c_{(k-1) / 2}
$$

for some $c_{(k-1) / 2}>0$. Let us also show that $r_{k} \rightarrow 0$. Indeed, since $\left.\tilde{u}_{n, k}\right|_{A_{r_{k-1} / r_{k}, 1 / r_{k}}} \rightarrow \tilde{u}$ in $C_{l o c}^{2}(\Pi)$, where $\tilde{u}$ can be extended to a non-trivial sign-changing solution to $-\mathcal{M}_{\lambda, \Lambda}^{+} u=|u|^{p_{+}^{* *}-1} u$ in $B_{\bar{r}}$, with homogenous Dirichlet boundary condition if $\Pi=\bar{B}_{\bar{r}} \backslash\{0\}$, or $-\mathcal{M}_{\lambda, \Lambda}^{+} u=|u|^{p_{+}^{* *}-1} u$ in $\mathbb{R}^{N}$ if $\Pi=\mathbb{R}^{N} \backslash\{0\}$. Now, in view of Proposition 3.4 the first case cannot occur and and thus we infer that $r_{k} \rightarrow 0$. In particular, since $M_{k-1} r_{k}^{\frac{2}{p_{n}-1}} \rightarrow \bar{M}$, it follows that $M_{k-1} \rightarrow+\infty$ and from (9.2) we infer that $M_{k} \rightarrow+\infty$.

Moreover, since $M_{k} s_{k}^{\frac{2}{p_{n}-1}}$ is bounded from above (by arguing as in the first part of Proposition 4.4) and $M_{k} \rightarrow+\infty$, then $s_{k} \rightarrow 0$.

To conclude the proof it remains to show that $u_{n} \rightarrow 0$ in $C_{l o c}^{2}(\bar{B} \backslash\{0\})$. The proof of this fact is identical to that of Proposition 7.4 with minor modifications. In particular, taking into account that $r_{k}, s_{k} \rightarrow 0$ and $u_{n}$ is negative in the last nodal component, then, for any fixed $\rho \in(0,1)$ and for all sufficiently large $n$ we obtain

$$
\left\|u_{n}\right\|_{\infty, A_{\rho}}=\left\|u_{n}^{-}\right\|_{\infty, A_{\rho}} \leq \frac{C}{\left(\frac{\rho^{2}}{2}\right)^{\frac{\tilde{N}_{-}-2}{2}}} r_{k}^{\tilde{N}_{-}-2-\frac{2}{p_{n}-1}},
$$

for some positive constant $C=C(N, \lambda, \Lambda)$ independent on $n$. This completes the proof when $k+1$ is even. 
Case 2: if $k+1$ is odd we consider the restriction to $B$ of the rescaled function

$$
\tilde{u}_{n, k}(x):=r_{k}^{\frac{2}{p_{n}-1}} u_{n}\left(r_{k} x\right), \quad x \in B_{1 / r_{k}},
$$

which is a solution to (1.10) having $k$ nodal regions, with $k$ even. Hence, from the inductive hypothesis, up to a subsequence, as $n \rightarrow+\infty$, we get that $M_{0} r_{k}^{\frac{2}{p_{n}-1}} \rightarrow+\infty, \ldots, M_{k-1} r_{k}^{\frac{2}{p_{n}-1}} \rightarrow+\infty$ and thus we infer that $M_{0} \rightarrow+\infty, \ldots, M_{k-1} \rightarrow+\infty$. Exploiting again the inductive hypothesis, we have $\frac{r_{i}}{r_{k}} \rightarrow 0, \frac{s_{i}}{r_{k}} \rightarrow 0$ for $i=1, \ldots, k-1$, which implies that $r_{i} \rightarrow 0, s_{i} \rightarrow 0$, for $i=1, \ldots, k-1$. Moreover $\frac{M_{2 j}}{M_{2 j+1}}=\frac{M_{2 j} r_{k}^{\frac{p_{n}-1}{2}}}{M_{2 j+1} r_{k}^{\frac{p_{n-1}}{2}}} \rightarrow c_{j}$ for $j=0, \ldots, \frac{k-2}{2}$ and $c_{j}$ positive constants, $\frac{M_{2 j+1}}{M_{2 j+2}}=\frac{M_{2 j+1} r_{k} \frac{p_{n}-1}{2}}{M_{2 j+2} r_{k}^{\frac{p p-1}{2}}} \rightarrow+\infty$, for $j=0, \ldots, \frac{k-4}{2}$.

Repeating exactly the same arguments of Sect. 8 , in the case of three nodal regions, we infer that $r_{k}, s_{k} \rightarrow 0, \frac{M_{k-1}}{M_{k}} \rightarrow+\infty$ and that $M_{k} \rightarrow \bar{M}$, where $\bar{M}$ is a positive constant. From this we also deduce that $u_{n}$ converges in $C_{l o c}^{2}(\bar{B} \backslash\{0\})$ to the unique positive solution of (1.11). The proof is complete.

\section{ENERGY OF SOLUTIONS}

Let $\Omega$ be a bounded radial domain in $\mathbb{R}^{N}$, i.e. $\Omega$ is either a ball or an annulus centered at the origin. Then the radial coordinate $r$ will belong either to $[0, R), R>0$, if $\Omega$ is the ball $B_{R}$, or to the interval $(a, b), 0<a<b$, if $\Omega$ is the annulus $A_{a, b}$.

We consider the space of radial functions in $\Omega$ which have constant sign and change convexity only once. More precisely we define

$$
\begin{aligned}
X_{\Omega}:= & \left\{u \in C_{r a d}^{2}(\bar{\Omega}) ;|u|>0 \text { and } \exists \varrho=\varrho(u) \in(a, b) \text { [resp. } \varrho \in(0, R) \text { if } \Omega\right. \text { is a ball] } \\
& \text { such that } u^{\prime \prime}(\varrho)=0, u^{\prime \prime}(r)<0 \text { for } r \in(a, \varrho) \text { and } u^{\prime \prime}(r)>0 \text { for } r \in(\varrho, b), \\
& \text { or } u^{\prime \prime}(r)>0 \text { for } r \in(a, \varrho) \text { and } u^{\prime \prime}(r)<0 \text { for } r \in(\varrho, b), \\
& {[\text { resp. } r \in(0, \varrho) \text { and } r \in(\varrho, R) \text { if } \Omega \text { is a ball] }\} . }
\end{aligned}
$$

Next, for an exponent $p>1$ and for any function $u \in X_{\Omega}$ we consider the radial weight:

$$
g_{u, p}(x):= \begin{cases}{[\varrho(u)]^{\gamma(p)}} & \text { if }|x|=r \leq \varrho(u), \\ |x|^{\gamma(p)} & \text { if }|x|>\varrho(u),\end{cases}
$$

with $\gamma(p):=2\left(\frac{p+1}{p-1}\right)-N$, and define the weighted energy

$$
E_{p, \Omega}(u):=\int_{\Omega}|u(x)|^{p+1} g_{u, p}(x) d x .
$$

It is elementary to check that $E_{p, \Omega}$ is invariant under the scaling $u_{\alpha}(x)=\alpha u\left(\alpha^{\frac{p-1}{2}} x\right)$ (see [4, Proof of Thorem 1.2]), i.e.

with $\Omega_{\alpha}=\alpha^{-\frac{p-1}{2}} \Omega$.

$$
E_{p, \Omega}(u)=E_{p, \Omega_{\alpha}}\left(u_{\alpha}\right)
$$

We observe that if $u$ is a solution of (1.1) with $k$ nodal regions, then the restrictions $u^{m}$ to each nodal region $\Omega^{m}$, for $m=1, \ldots, k$, belong to the space $X_{\Omega^{m}}$. Therefore we can consider the energy of each function $u^{m}$ in the corresponding nodal region $\Omega^{m}$ :

$$
E_{p, \Omega^{m}}\left(u^{m}\right):=\int_{\Omega^{m}}\left|u^{m}(x)\right|^{p+1} g_{u^{m}, p}(x) d x, \text { for } m=1, \ldots, k,
$$


and define the total energy of the solution $u$ in the ball $B$ as

$$
E_{p}^{T}(u):=\sum_{m=1}^{k} E_{p, \Omega^{m}}\left(u^{m}\right)
$$

A similar energy can be defined for any positive (fast decaying) radial solution $U_{ \pm}$of the critical equation in $\mathbb{R}^{N}$

We denote it by $E^{*}\left(U_{ \pm}\right)$, i.e.

$$
-\mathcal{M}_{\lambda, \Lambda}^{ \pm}\left(D^{2} u\right)=u^{p_{ \pm}^{*}} \text { in } \mathbb{R}^{N} .
$$

$$
E^{*}\left(U_{ \pm}\right):=\int_{\mathbb{R}^{N}}\left|U_{ \pm}(x)\right|^{p_{ \pm}^{*}+1} g_{U_{ \pm}}^{*}(x) d x
$$

where

$$
g_{U_{ \pm}}^{*}(x):= \begin{cases}{\left[\varrho\left(U_{ \pm}\right)\right]^{\gamma^{*}}} & \text { if }|x|=r \leq \varrho\left(U_{ \pm}\right) \\ |x|^{\gamma^{*}} & \text { if }|x|=r>\varrho\left(U_{ \pm}\right)\end{cases}
$$

with $\gamma^{*}:=2\left(\frac{p_{ \pm}^{*}+1}{p_{ \pm}^{*}-1}\right)-N$.

Note that, by the invariance of the energy with respect the usual scaling, $\Sigma_{ \pm}^{*}:=E^{*}\left(U_{ \pm}\right)$is a constant depending only on $\lambda, \Lambda, N$.

We now prove Theorem 1.3 (we refer to Theorem 1.2 for the notations).

Proof of Theorem 1.3. Let us first consider the restriction of the solution $u_{\varepsilon}$ to the first nodal region $\Omega_{\varepsilon}^{1}=B_{r_{1}}$. We denote it by $u_{\varepsilon}^{1}$. The function

$$
\tilde{u}_{\varepsilon}^{1}(x):=r_{1}^{\frac{2}{p_{\varepsilon}-1}} u_{\varepsilon}^{1}\left(r_{1} x\right), \quad x \in B,
$$

is the unique positive solution of

$$
\left\{\begin{array}{cl}
-\mathcal{M}_{\lambda, \Lambda}^{-}\left(D^{2} u\right)=u^{p_{\varepsilon}} & \text { in } B, \\
u=0 & \text { on } \partial B .
\end{array}\right.
$$

By the scaling invariance (10.3), we have

$$
E_{p_{\varepsilon}, \Omega_{\varepsilon}^{1}}\left(u_{\varepsilon}^{1}\right)=E_{p_{\varepsilon}, B}\left(\tilde{u}_{\varepsilon}^{1}\right) .
$$

By 4. Theorem 1.2] we immediately deduce that, as $\varepsilon \rightarrow 0$,

$$
E_{p_{\varepsilon}, \Omega_{\varepsilon}^{1}}\left(u_{\varepsilon}^{1}\right) \rightarrow \int_{\mathbb{R}^{N}}\left(U_{-}\right)^{p_{-}^{*}+1} g_{U_{-}}^{*} d x=\Sigma_{-}^{*} .
$$

This gives the first contribution to the limit of the total energy in (1.8).

On the other hand we recall that the nodal radii $r_{1}, r_{2}, \ldots, r_{k-1}$ converge respectively to 0 , $\bar{r}_{2}, \ldots, \bar{r}_{k-1}$, where $\bar{r}_{2}, \ldots, \bar{r}_{k-1}$ are the nodal radii of the limit function $\bar{u}$ given by Theorem 1.2

Thus, by the convergence of $u_{\varepsilon} \rightarrow \bar{u}$ in $C_{l o c}^{2}(\bar{B} \backslash\{0\})$ we have that the restriction $u_{\varepsilon}^{m}$ of $u_{\varepsilon}$ to its nodal region $\Omega_{\varepsilon}^{m}, m=2, \ldots, k$, converges to the restriction of $\bar{u}$ to the corresponding nodal region, i.e.:

$$
u_{\varepsilon}^{2} \rightarrow \bar{u}_{1}, \ldots, u_{\varepsilon}^{m} \rightarrow \bar{u}_{m-1} .
$$

Then, using also that $M_{i} \rightarrow \bar{M}_{i}$, we have

$$
\begin{aligned}
E_{p_{\varepsilon}, \Omega_{\varepsilon}^{2}}\left(u_{\varepsilon}^{2}\right) & =\int_{\Omega_{\varepsilon}^{2}}\left|u_{\varepsilon}^{2}\right|^{p_{\varepsilon}+1} g_{u_{\varepsilon}^{2}} d x \rightarrow \int_{B_{\bar{r}_{2}}}\left|\bar{u}_{1}\right|^{p_{-}^{*}+1} g_{\bar{u}_{1}}^{*} d x \\
& \cdots \\
E_{p_{\varepsilon}, \Omega_{\varepsilon}^{m}}\left(u_{\varepsilon}^{m}\right) & =\int_{\Omega_{\varepsilon}^{m}}\left|u_{\varepsilon}^{m}\right|^{p_{\varepsilon}+1} g_{u_{\varepsilon}^{m}} d x \rightarrow \int_{A_{\bar{r}_{m-1}, \bar{r}_{m}}}\left|\bar{u}_{m}\right|^{p_{-}^{*}+1} g_{\bar{u}_{m-1}}^{*} d x,
\end{aligned}
$$


where $\bar{r}_{m}=1$ if $m=k$. Thus the assertion (1.8) holds and the proof is complete.

We now study the limit energy of a family of sign-changing solution to (1.10) having $k$ nodal regions, as $n \rightarrow+\infty$ and prove Theorem 1.5. We denote by $E^{* *}\left(W_{-}\right)$the energy of the only radial positive fast decaying solution to (1.12), namely

$$
E^{* *}\left(W_{-}\right)=\int_{\mathbb{R}^{N} \backslash B}\left|W_{-}(x)\right|^{p_{+}^{* *}+1} g_{W_{-}}^{* *}(x) d x
$$

where

$$
g_{W_{-}}^{* *}(x):= \begin{cases}{\left[\varrho\left(W_{-}\right)\right]^{\gamma^{* *}}} & \text { if } 1 \leq|x|=r \leq \varrho\left(W_{-}\right), \\ |x|^{\gamma^{* *}} & \text { if } \quad|x|=r>\varrho\left(W_{-}\right),\end{cases}
$$

with $\gamma^{* *}:=2\left(\frac{p_{+}^{* *}+1}{p_{+}^{* *}-1}\right)-N$. Since $W_{-}$is fast decaying easily we have:

Lemma 10.1. The energy $E^{* *}\left(W_{-}\right)$is finite.

Proof. Since $W_{-}$is fast decaying we can find $C>0$ and $\bar{r}>1$ such that

$$
W_{-}(r) \leq \frac{C}{r^{\tilde{N}_{-}-2}} \text { for } r>\bar{r}
$$

Up to choosing a larger $\bar{r}$ we can assume without loss of generality that $\bar{r}>\varrho\left(W_{-}\right)$and thus

$$
\begin{aligned}
E^{* *}\left(W_{-}\right)= & \int_{\mathbb{R}^{N} \backslash B}\left|W_{-}(x)\right|^{p_{+}^{* *}+1} g_{W_{-}}^{* *}(x) d x \\
= & \int_{1}^{\varrho\left(W_{-}\right)}\left|W_{-}(r)\right|^{p_{+}^{* *}+1}\left[\varrho\left(W_{-}\right)\right]^{2\left(\frac{p_{+}^{* *+1}}{p_{+}^{* *-1}}\right)-N} r^{N-1} d r \\
& +\int_{\varrho\left(W_{-}\right)}^{+\infty}\left|W_{-}\right|_{+}^{p_{+}^{* *}+1} r^{2\left(\frac{p_{+}^{* *+1}}{p_{+}^{* *}-1}\right)-N^{N}} r^{N-1} d r \\
= & (I)+(I I) .
\end{aligned}
$$

Clearly $(I)$ is finite. For $(I I)$, exploiting (10.11) we have

$$
\begin{aligned}
(I I) & \leq \int_{\varrho\left(W_{-}\right)}^{\bar{r}}\left|W_{-}(r)\right|^{p_{+}^{* *}+1} r^{2\left(\frac{p_{+}^{* *}+1}{p_{+}^{*}-1}\right)-N} r^{N-1} d r+C \int_{\bar{r}}^{+\infty} r^{-\left(\tilde{N}_{-}-2\right)\left(p_{+}^{* *}+1\right)+2\left(\frac{p_{+*}^{* *}+1}{p_{+}^{* *}-1}\right)-1} d r \\
& =(I I I)+(I V) .
\end{aligned}
$$

Now, $(I I I)$ is finite and so is $(I V)$ by a straightforward computation because $p_{+}^{* *}>p_{-}^{*}>$ $\frac{\tilde{N}_{-}}{\tilde{N}_{-}-2}$.

We now prove Theorem 1.5 (for the notations we refer to Theorem 1.4 and the beginning of this section).

Proof of Theorem 1.5. We argue by induction on $k \geq 2$. We begin with the basic step $k=2$.

Assume that $u_{n}$ is a sign-changing solution to (1.10) having two nodal regions and let $r_{1}$ be the node of $u_{n}$. Then $\Omega_{n}^{1}=B_{r_{1}}$ and $\Omega_{n}^{2}=A_{r_{1}, 1}$. We consider the rescaled function $\tilde{u}_{n}$ defined in (7.2). By construction $\left.\tilde{u}_{n}^{+}\right|_{B}$ coincides with the unique positive solution of (1.10), i.e. $\left.\tilde{u}_{n}^{+}\right|_{B}=v_{p_{n},+}$ and it is uniformly bounded (see Proposition (7.2). Hence $\left.\tilde{u}_{n}^{+}\right|_{B} \rightarrow \bar{v}$ in $C^{2}(\bar{B})$, where $\bar{v}$ is the unique positive solution of (1.11). Hence, exploiting the scaling invariance of the energy and passing to the limit as $n \rightarrow+\infty$ we get that

$$
E_{p_{n}, \Omega_{n}^{1}}\left(u_{n}^{1}\right)=E_{p_{n}, B}\left(\tilde{u}_{n}^{+}\right)=\int_{B}\left|\tilde{u}_{n}^{+}\right|^{p_{n}+1} g_{\tilde{u}_{n}^{+}} d x \rightarrow \int_{B}|\bar{v}|^{p_{+}^{* *}+1} g_{\bar{v}} d x=E_{p_{+}^{* *}, B}(\bar{v}) .
$$


For $u_{n}^{2}$ we have $\Omega_{n}^{2}=A_{r_{1}, 1}$ and exploiting the scaling invariance and the definition of the energy we have

$$
\begin{aligned}
E_{p_{n}, \Omega_{n}^{2}}\left(u_{n}^{2}\right)=E_{p_{n}, A_{1,1 / r_{1}}}\left(\tilde{u}_{n}^{-}\right) & =\int_{A_{1, \tilde{t}_{1}}}\left|\tilde{u}_{n}^{-}\right|^{p_{n}+1}\left[\tilde{t}_{1}\right]^{\gamma\left(p_{n}\right)} d x+\int_{A_{\tilde{t}_{1}, 1 / r_{1}}}\left|\tilde{u}_{n}^{-}\right|^{p_{n}+1}|x|^{\gamma\left(p_{n}\right)} d x, \\
& =(I)+(I I),
\end{aligned}
$$

where $\tilde{t}_{1}=\varrho\left(\tilde{u}_{n}^{-}\right)$. From Proposition 7.3 we have $\left.\tilde{u}_{n}^{-}\right|_{A_{1 / 1,1}} \rightarrow W_{-}$in $C_{\text {loc }}^{2}\left(\mathbb{R}^{N} \backslash B\right)$, where $W_{-}$ is the unique radial positive fast decaying solution of (1.12). We claim that we can pass to limit under the integral sign in $(I)$ and $(I I)$. Indeed, for $(I)$ it is obvious because $\tilde{u}_{n}$ is uniformly bounded and $\tilde{t}_{1} \rightarrow \bar{t}_{1} \in(0,+\infty)$, where $\bar{t}_{1}=\varrho\left(W_{-}\right)$, while for $(I I)$, taking into account (7.7) and that $p_{n}>p_{-}^{*}>\frac{\tilde{N}_{-}}{N_{--2}}$, we easily obtain

$$
\left|\tilde{u}_{n}^{-}(r)\right|^{p_{n}+1} r^{\frac{p_{n}+1}{p_{n}-1}-N} r^{N-1} \leq C r^{-\left(\tilde{N}_{-}-2\right)\left(p_{n}+1\right)+2 \frac{p_{n}+1}{p_{n}-1}-1} \leq C r^{-1-\delta} \text { for } r \geq \tilde{t}_{1},
$$

for some $C>0, \delta>0$ independent on $n$. Hence, by Lebesgue's dominated convergence theorem we can pass to the limit under the integral sign in (II) and thus we conclude that

$$
\begin{aligned}
\lim _{n \rightarrow+\infty} E_{p_{n}, \Omega_{n}^{2}}\left(u_{n}^{2}\right) & =\int_{A_{1, \bar{t}_{1}}}\left|W_{-}(x)\right|^{p_{+}^{* *}+1}\left[\bar{t}_{1}\right]^{\gamma\left(p_{+}^{* *}\right)} d x+\int_{\mathbb{R}^{N} \backslash B_{\bar{t}_{1}}}\left|W_{-}(x)\right|^{p_{+}^{* *}+1}|x|^{\gamma\left(p_{+}^{* *}\right)} d x \\
& =E^{* *}\left(W_{-}\right)=\Sigma_{+}^{* *} .
\end{aligned}
$$

Combining (10.12) and (10.13) we complete the proof of the basic step.

Now let us prove the inductive step. Let $u_{n}$ be a radial solution to (1.10) with $k+1$ nodal regions and consider the rescaled function $\tilde{u}_{k, n}$ defined by (9.3). Clearly, by invariance under this scaling and by definition we easily have

$$
E_{p_{n}}^{T}\left(u_{n}\right)=E_{p_{n}}^{T}\left(\left.\tilde{u}_{k, n}\right|_{B}\right)+E_{p_{n}, A_{1,1 / r_{k}}}\left(\left.\tilde{u}_{k, n}\right|_{A_{1,1 / r_{k}}}\right) .
$$

Since the restriction $\left.\tilde{u}_{k, n}\right|_{B}$ is a radial sign-changing solution of (1.10) with $k$ nodal regions then by the induction hypothesis we infer that

$$
\lim _{n \rightarrow+\infty} E_{p_{n}}^{T}\left(\left.\tilde{u}_{k, n}\right|_{B}\right)= \begin{cases}\frac{k}{2} E_{p_{+}^{* *}, B}(\bar{v})+\frac{k}{2} \Sigma_{+}^{* *} & \text { if } k \text { is even, } \\ \frac{k+1}{2} E_{p_{+}^{* *}, B}(\bar{v})+\frac{k-1}{2} \Sigma_{+}^{* *} & \text { if } k \text { is odd. }\end{cases}
$$

For the second term of (10.14), if $k$ is even then $u_{n}$ has an odd number of nodal components and by Theorem 1.4 we have $r_{k} \rightarrow 0, u_{n}^{k+1}=\left.u_{n}\right|_{A_{r_{k}, 1}}$ is uniformly bounded and $u_{n}^{k+1} \rightarrow \bar{v}$ in $C_{l o c}^{2}(\bar{B} \backslash\{0\})$. Therefore, by the usual invariance under scaling and exploiting these properties we get that

$$
\lim _{n \rightarrow+\infty} E_{p_{n}, A_{1,1 / r_{k}}}\left(\left.\tilde{u}_{k, n}\right|_{A_{1,1 / r_{k}}}\right)=\lim _{n \rightarrow+\infty} E_{p_{n}, A_{r_{k}, 1}}\left(u_{n}^{k+1}\right)=E_{p_{+}^{* *}, B}(\bar{v}) .
$$

If $k$ is odd, then applying Theorem 1.4 to $\left.\tilde{u}_{k, n}\right|_{B}$ we infer that $\left.\tilde{u}_{k, n}\right|_{B} \rightarrow \bar{v}$ in $C_{l o c}^{2}(\bar{B} \backslash\{0\})$. Hence, arguing as in the proof of Proposition 7.3 we have $\left.\tilde{u}_{n}^{-}\right|_{A_{1,1 / r_{k}}} \rightarrow W_{-}$in $C_{\text {loc }}^{2}\left(\mathbb{R}^{N} \backslash B\right)$ and $\tilde{t}_{k} \rightarrow \bar{t}_{k} \in(1,+\infty), \bar{t}_{k}=\varrho\left(W_{-}\right)$. Then, as in the proof of the basic step $k=2$ and exploiting an analogous uniform upper bound (the proof is the same as that of Proposition 7.3 with minor changes), we get that

$$
\lim _{n \rightarrow+\infty} E_{p_{n}, A_{1,1 / r_{k}}}\left(\left.\tilde{u}_{k, n}\right|_{A_{1,1 / r_{k}}}\right)=\Sigma_{+}^{* *} .
$$

At the end, combining (10.14)-(10.17) we obtain (1.14). 


\section{REFERENCES}

[1] Atkinson, F.V., Brezis, H., Peletier, L.A., Nodal solutions of elliptic equations with critical Sobolev exponents, Journal of Diff. Eq. (1990), 85 (1), 151-170.

[2] Atkinson, F.V., Peletier, L.A., Emden-Fowler equations involving critical exponents, Nonlinear Anal. Theory Methods Appl. (1986), 10 (8), 755-776.

[3] M. Ben Ayed, K. El Mehdi, F. Pacella, Classification of low energy sign-changing solutions of an almost critical problem, Journal of Funct. Analysis (2007), 250, 347-373.

[4] I. Birindelli, G. Galise, F. Leoni, F. Pacella, Concentration and energy invariance for a class of fully nonlinear elliptic equations, Calc. Var. PDE. (2018), 57: 158.

[5] J. Busca, M. J. Esteban, A. Quaas, Nonlinear eigenvalues and bifurcation problems for Pucci's operators, Ann. Inst. H. Poincaré Anal. Non Linéaire 22 (2005), no. 2, 187-206.

[6] F. Da Lio, B. Sirakov, Symmetry properties of viscosity solutions to nonlinear uniformly elliptic equations, J. Eur. Math. Soc. (2007), 9, 317-330.

[7] F. De Marchis, I. Ianni, F. Pacella, A Morse index formula for radial solutions of Lane-Emden problems, Adv. Math. (2017), 322, 682-737.

[8] M. J. Esteban, P. L. Felmer, A. Quaas, Eigenvalues for radially symmetric fully nonlinear operators, Comm. Partial Differential Equations (2010), 35 no. 9, 1716-1737.

[9] P. L. Felmer, A. Quaas, On critical exponents for the Pucci's extremal operators, Ann. Inst. H. Poincaré Anal. Non Linéaire (2003), 20 (5), 846-865.

[10] G. Galise, F. Leoni e F. Pacella, Existence results for fully nonlinear equations in radial domains, Commun. Partial Differential Equations (2017), 42:5, 757-779

[11] G. Galise, A. Iacopetti, F. Leoni, Liouville-type results in exterior domains for radial solutions of fully nonlinear equations (preprint) arXiv:1907.12985

[12] A. Iacopetti, Asymptotic analysis for radial sign-changing solutions of the Brezis-Nirenberg problem, Annali di Matematica Pura ed Applicata (2015), Vol. 194 Issue 6, 1649-1682.

[13] A. Iacopetti, F. Pacella, Asymptotic analysis for radial sign-changing solutions of the Brezis-Nirenberg problem in low dimensions, Progress in Nonlinear Diff. Eq. and their Appl. (2015), Vol. 86, 325-343.

[14] A. Iacopetti, G. Vaira, Sign-changing tower of bubbles for the Brezis-Nirenberg problem, Commun. Contemp. Math. (2016), 18, 1550036.

[15] A. Iacopetti, G. Vaira, Sign-changing blowing-up solutions for the Brezis-Nirenberg problem in dimensions four and five, Annali della Scuola Normale Superiore di Pisa (2018), Vol. XVIII, Issue 1, 1-38.

[16] A. Quaas, B. Sirakov, Existence results for nonproper elliptic equations involving the Pucci operator, Comm. Partial Differential Equations 31 (2006), no. 7-9, 987-1003.

Dipartimento di Matematica, Sapienza Università di Roma, P.le Aldo Moro 2, 00185 Roma, Italy

E-mail address: galise@mat.uniroma1.it (G. Galise),

E-mail address: iacopetti@mat.uniroma1.it (A. Iacopetti),

E-mail address: leoni@mat.uniroma1.it (F. Leoni),

E-mail address: pacella@mat.uniroma1.it (F. Pacella). 